\title{
The Connections of the Inferior Colliculus and the Organization of the Brainstem Auditory System in the Greater Horseshoe Bat (Rhinolophus ferrumequinum)
}

\author{
HERMANN SCHWEIZER \\ Arbeitskreis Neuro- und Rezeptorphysiologie, Fachbereich Biologie (Zoologie), J.W. \\ Goethe Universitaet, D 6000 Frankfurt/M Federal Republic of Germany
}

\begin{abstract}
The connections of the inferior colliculus, the mammalian midbrain auditory center, were determined in the greater horseshoe bat (Rhinolophus ferrumequinum), using the horseradish peroxidase method. In order to localize the auditory centers of this bat, brains were investigated with the aid of cell and fiber-stained material.

The results show that most auditory centers are highly developed in this echolocating bat. However, the organization of the central auditory system does not generally differ from the mammalian scheme. This holds also for the organization of the superior olivary complex where a well-developed medial superior olivary nucleus was found. In addition to the ventral and dorsal nuclei of the lateral lemniscus a third well-developed nucleus has been defined which projects ipsilaterally to the inferior colliculus and which was called the intermediate nucleus of the lateral leminiscus.

All nuclei of the central auditory pathway project ipsi-, contra-, or bilaterally to the central nucleus of the inferior colliculus with the exception of the medial nucleus of the trapezoid body and the medial geniculate body. The tonotopic organization of these projections and their possible functions are discussed in context with neurophysiological investigations.
\end{abstract}

Insectivorous bats are mammals that hunt at night with the help of an active sonar system. They emit ultrasonic calls and extract from the returning echoes information about their environment and their prey with the aid of a highly developed auditory system. Since the discovery of this specialized orientation system in bats many neurophysiological experiments have been performed on acoustic processing in the central auditory system of these animals. The neurophysiological data show that the auditory system of echolocating bats is highly specialized and adapted to their specific orientation behavior (e.g., Neuweiler, '80; Pollack, '80; Suga and O'Neill, '80).

Surprisingly, only a few anatomical data exist for the central auditory system in bats (for review see Henson, '70). Most anatomical investigations show that some of the auditory centers are highly developed. But, two main questions arise from earlier investigations: How is the auditory pathway organized up to the inferior colliculus and how well-developed are the higher auditory centers, the medial geniculate body, and the auditory cortex?

The most detailed anatomical study of the auditory system in bats is the early work of Poljak ('26a,b) in Rhinolophus ferrumequinum and Nyctalus noctula. Poljak concluded that the organization of the central auditory pathway in these bats does not differ significantly from that of other mammals. However, his studies of fiber connections were done only with myelin-stained material.

In contrast to Poljak's findings, Harrison and Irving ('66), Irving and Harrison ('67), and Masterton and Diamond ('67) emphasized some differences in the central auditory system of bats, especially concerning the organization of the superior olivary complex. They advocated that bats do not have a medial superior olivary nucleus. Therefore it was generally agreed that the organization of the auditory system of echolocating bats differed from that of other mammals, and not until the auditory pathways of bats were recently investigated 
with modern neuroanatomical tracing techniques (Schweizer, '78; Schweizer and Radtke, '80; Zook, '79; Zook and Casseday, '78; Zook and Casseday, ' 80 ) did this concept begin to change.

This paper presents data on the structure of the lower auditory centers and the connections of the inferior colliculus of the greater horseshoe bat, Rhinolophus ferrumequinum, and tries to clarify the question of whether there are fundamental differences in the anatomical organization of the central auditory system of echolocating bats in comparison to that of other mammals.

\section{METHODS}

In order to examine the cytoarchitecture of the auditory centers of the greater horseshoe bat, six brains were fixed in formalin, embedded in paraplast or celloidin, and cut in the

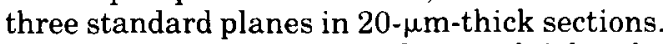
Sections were stained with cresylviolet for cells, with hematoxyline for myelinated fibers, or with cresylviolet and luxol fast blue for a combined cell and fiber stain. For visualization of unmyelinated fibers, some sections were processed according to a modified Bodian silver impregnation method (Ziesmer, '52).

The connections of the inferior colliculus were studied with the horseradish peroxidase (HRP) method. The animals were anesthetized by intraperitoneal injection of nembutal (2.5-3.0 mg/100 gm body weight) and placed in a head holder. The skin and muscles over the midbrain were removed and a small hole was drilled into the skull. HRP (Sigma type VI or HRP from Serva) was injected into different parts of the IC either by pressure or iontophoretically. In five cases HRP was applied in solid form.

Survival times varied from 4 to 96 hours, but in most experiments the survival time was 24 hours. The animals were then deeply anesthetized with nembutal $(4 \mathrm{mg} / 100 \mathrm{gm}$ body weight) and perfused through the heart with phosphate-buffered heparinized saline $(\mathrm{pH} 7.2)$ followed by the fixative solution. In most animals the fixative consisted of $2 \%$ paraformaldehyde $+1 \%$ glutaraldehyde in $0.05 \mathrm{M}$ phosphate buffer $(\mathrm{pH} 7.2$ ). After 20-30 minutes of perfusion the brains were removed from the skull, covered with a thin layer of egg yolk, and postfixed for 4-6 hours in the cold. The tissue was then washed overnight in two changes of cold $0.1 \mathrm{M}$ phosphate buffer (pH 7.2) containing $20 \%$ sucrose. The following day the brains were cut on a freezing microtome into $40-60-\mu \mathrm{m}$-thick frontal sections. The sections were collected in cold $0.1 \mathrm{M}$ phosphate buffer (pH 7.2) and were processed the same day for the HRP reaction.

Three different procedures for HRP reaction were used: The DAB method (Graham and Karnovsky, '66), the DAB cobalt chloride method (Adams, '77), and the TMB method (Mesulam, '78). After washing, the sections were mounted on gelatinized slides, air dried, counterstained in cresylviolet (for DAB-reacted sections) or in a $1 \%$ acidified neutral red solution (for TMB reacted sections), dehydrated in graded ethanol, cleared in xylene, and coverslipped.

The sections were examined with a Zeiss photomicroscope under brightfield or darkfield illumination or between crossed polarizers (for the TMB method) (Illing and Waessle, '79). Drawings were made from frontal sections to show the cytoarchitecture of auditory nuclei and to show the spatial distribution of labeled cells.

\begin{tabular}{ll}
\hline & Abbreviations \\
ALPO & Anterolateral periolivary nucleus \\
AVCN & Anteroventral cochlear nuclues \\
BIC & Brachium of the inferior colliculus \\
CGM & Central gray matter \\
CN & Cochlear nucleus \\
DCN & Dorsal cochlear nucleus \\
DNLL & Dorsal nucleus of the lateral lemniscus \\
DPO & Dorsal periolivary nucleus \\
IC & Inferior colliculus \\
ICC & Central nucleus of the inferior colliculus \\
ICP & Pericentral nucleus of the inferior colliculus \\
ICX & External nucleus of the interior colliculus \\
INLL & Intermediate nucleus of the lateral lemniscus \\
LL & Lateral lemniscus \\
LNTB & Lateral nucleus of the trapezoid body \\
LSO & Lateral superior olivary nucleus \\
MGB & Medial geniculate body \\
MNTB & Medial nucleus of the trapezoid body \\
MSO & Medial superior olivary nucleus \\
PVCN & Posteroventral cochlear nucleus \\
SOC & Superior olivary complex \\
VMPO & Ventromedial periolivary nucleus \\
VNLL & Ventral nucleus of the lateral lemniscus \\
VNTB & Ventral nucleus of the trapezoid body \\
VOP & Ventral periolivary nucleus \\
\end{tabular}

\section{RESULTS}

The presentation of the results is divided into two parts. Part one gives a brief description of the cytoarchitecture of the auditory centers with the exception of the medial geniculate body and the auditory cortex. A detailed description of subdivisions of different auditory nuclei and the neurons composing these 


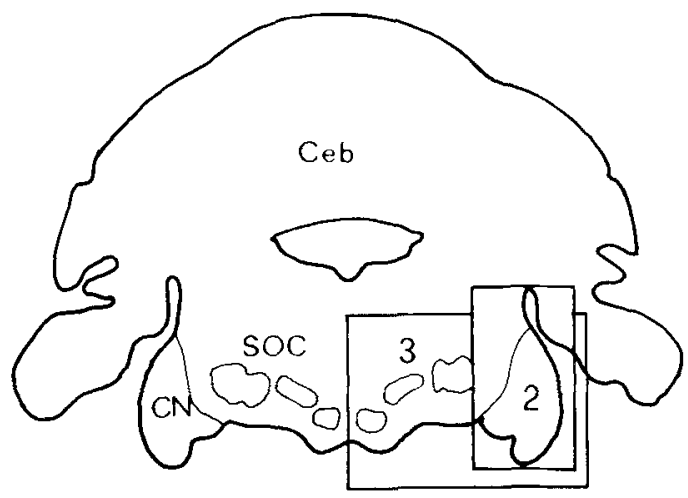

Fig. 1. Frontal sections of the medulla oblongata and the overlying cerebellum (Ceb) (left) and of the midbrain (right) showing the position of the lower auditory centers.

nuclei will be part of further studies. In part two, the results of the HRP experiments are presented.

\section{Part I: Cytoarchitecture of the lower auditory centers}

Figure 1 shows the position of the lower auditory centers in two frontal sections of the brainstem. The description of these centers starts from caudal to rostral with the cochlear nucleus complex followed by the superior olivary complex, the nuclei of the lateral lemniscus, and the inferior colliculus.

The cochlear nucleus complex. As in other mammals the cochlear nucleus complex (CN) of Rhinolophus ferrumequinum can be divided on the basis of cytoarchitectural features (cell size, cell shape, packing density of cells) into three subnuclei (Fig. 2): dorsal (DCN), posteroventral (PVCN), and anteroventral (AVCN) cochlear nucleus. The boundary between the DCN, th PVCN, and the AVCN is formed by a dense layer of small, darkly stained granular cells. The PVCN and the AVCN are clearly separated by the incoming fibers of the eighth nerve root. All three subnuclei can be divided into different subdivisions composed of different cell types.

The dorsal cochlear nucleus. The dorsal cochlear nucleus is the smallest of the three subnuclei of the cochlear nucleus complex. In cell-stained material, the DCN of Rhinolophus can be divided into a dorsocaudal and a ventral part. The boundary between the two DCN parts is made apparent by a shallow sulcus on

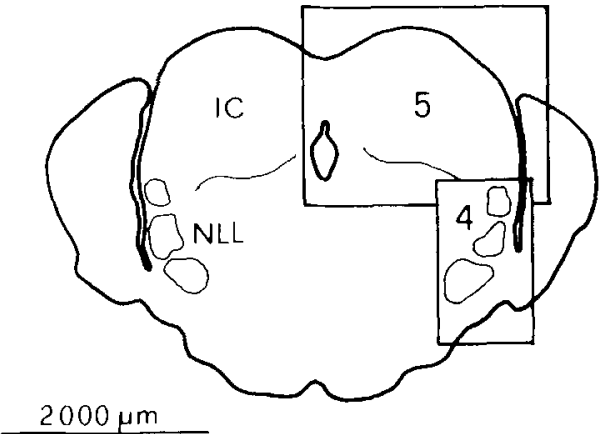

The rectangles in the right half of each section indicate the location of the sections in figures 2 to 5. Abbrev.: NLLnuclei of the lateral lemniscus.

the lateral brain surface (Fig. 2). In the ventral part a superficial ependymal cell layer is followed medially by a molecular cell layer, a "fusiform" cell layer, and a polymorphic cell region. However, the fusiform cell layer can be distinguished from the polymorphic cell region only by a more orderly arrangement of fusiform cells, which are often oriented with their long axis running from lateral to medial, and by a slightly higher density of granular cells. In contrast to the ventral DNC, the dorsocaudal region shows a disorderly cell arrangement. But the main difference is that a molecular layer is not developed in this part of the DCN.

The Posteroventral Cochlear Nucleus. The PVCN is situated ventral to the DCN and just caudal to the acoustic branch of the eighth nerve root. It will be divided in this study only into three subdivisions--a central, a lateral, and a ventral (Fig. 2).

In the ventral subdivision the neurons are generally smaller than in the other two regions and they are more densely packed. In the rostral third of the PVCN the ventral subdivision is separated from the lateral subdivision by a region composed of densely packed, small, round cells.

The lateral subdivision is mainly composed of large, spindle-shaped neurons which are arranged in an orderly manner with their long axes parallel to the outgoing fibers which contribute to the trapezoid body. The lateral and the ventral subdivision together have a convoluted "S" shape which gives the PVCN of Rhinolophus its typical form. 

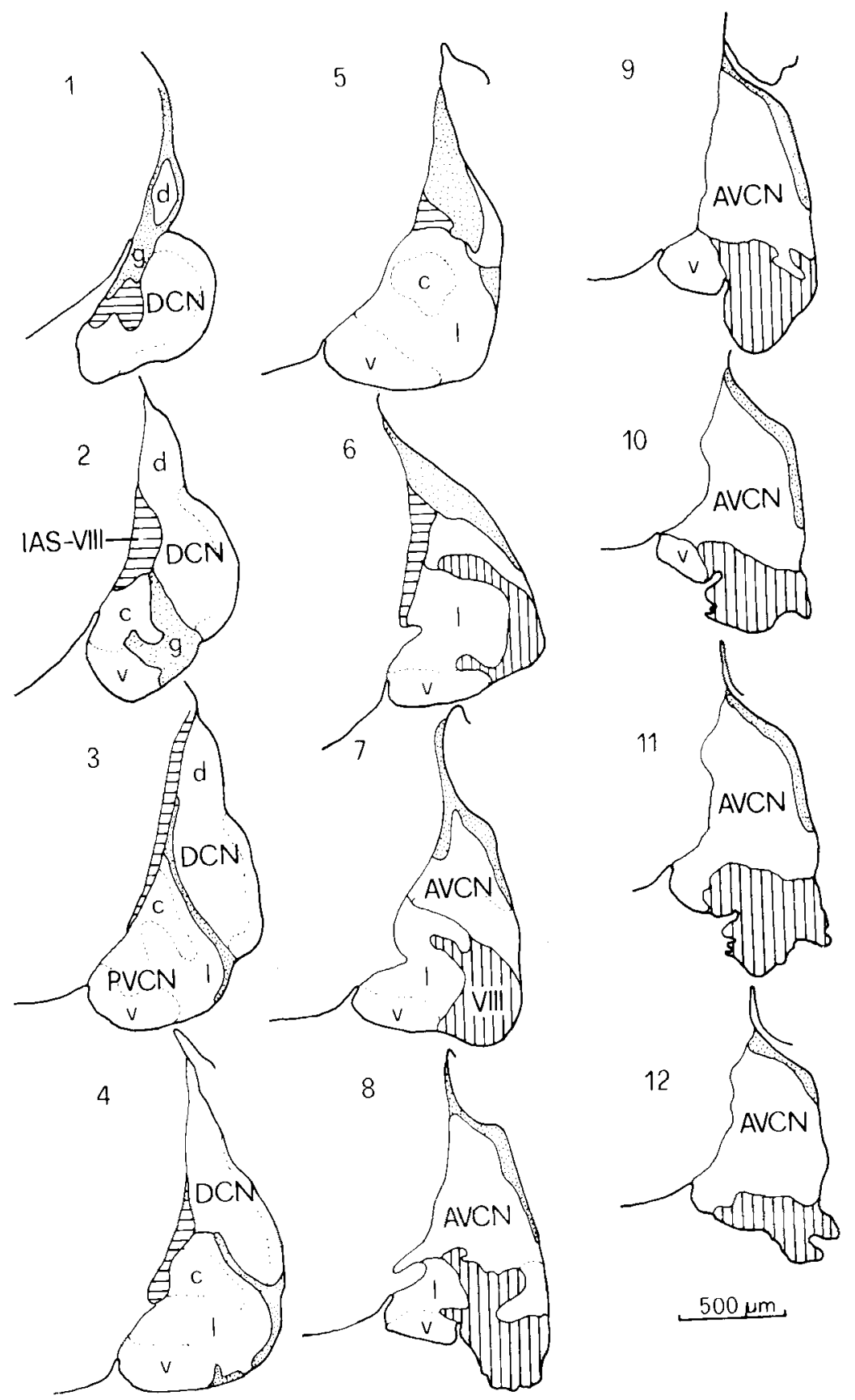

$500 \mu \mathrm{m}$

Fig. 2. Frontal sections of the cochlear nucleus complex. Section 1 is most caudal, section 12 most rostral. The sections are spaced at $80 \mu \mathrm{m}$. Abbrev.: d-dorsocaudal region of the DCN; g-granular cell region (dotted area in each section); IAS-VIII-fibers of the intermediate acoustic stria and of the vestibular nerve (horizontal hatching); $c$-central, v-ventral, l-lateral subdivision of the PVCN; VIII-acoustic nerve root (vertical hatching). 
The central subdivision is separated from the lateral subdivision by a region of very low cell density and many incoming cochlear fibers and it stands out by reason of its numerous large, faintly stained, ovoid and multipolar cells. The central subdivision may correspond in position to the "octopus cell area" of other mammals.

The Anteroventral Cochlear Nucleus. of the three subnuclei of the CN the anteroventral is by far the biggest. It is separated from the DCN by a thin granular cell layer which also covers the caudal two-thirds of the subnucleus laterally and dorsally (Fig. 2). The ventral border of the AVCN is formed by the incoming fibers of the acoustic nerve root. In cell-stained material the AVCN shows a more uniform structure than do the PVCN and the DCN, because one main cell type (mediumsized, round to ovoid) is common throughout the nucleus. Although the cell composition and especially the cell density vary considerably in different parts of the subnucleus, in this study the AVCN will not be further subdivided.

The Superior Olivary Complex. The superior olivary complex (SOC) of Rhinolophus ferrumequinum consists of three main nuclei surrounded by several smaller cell groups (Fig. 3). Although the cytoarchitecture differs in some respects from that in other mammals the nomenclature used in other mammals is adopted here. Therefore the three main nuclei are called lateral superior olivary nucleus (LSO), medial superior olivary nucleus (MSO), and medial nucleus of the trapezoid body (MNTB). The justification for the use of this nomenclature is based not only on cytoarchitectural features, but is also taken from the results of the HRP experiments, as will be shown later. The smaller cell groups of the SOC are divided into two main groups, the nuclei of the trapezoid body and the periolivary nuclei: ventral nucleus of the trapezoid body (VNTB), lateral nucleus of the trapezoid body (LNTB), ventral periolivary nucleus (VPO), ventromedial periolivary nucleus (VMPO), dorsal periolivary nucleus (DPO), and anterolateral periolivary nucleus (ALPO) (Fig. 3). However, in this report the description of the SOC nuclei is restricted to the three main nuclei.

The Lateral Superior Olivary Nucleus. The LSO is by far the largest of all nuclei of the SOC. Its first caudal neurons appear medial to the cochlear nucleus, lateral to the rostral pole of the motor nucleus of the facial nerve, just medioventral to the facial nerve root (Fig. 3). More rostrally, in the middle parts of the SOC, the nucleus has a convoluted shape with a dorsal and ventral hilus where the trapezoid body fibers enter the nucleus. The outline of the LSO is more rounded in its rostral parts and a sharp delineation against the surrounding periolivary nuclei becomes difficult. The nucleus mainly consists of medium-sized, spindleshaped neurons arranged with their long axes parallel to the convolutions of the nucleus. Some large multipolar cells, more numerous in lateral parts, are scattered throughout the nucleus.

The Medial Superior Olivary Nucleus. Out of all components of the SOC the medial nucleus shows the strongest divergence from the cytoarchitectural scheme of other mammals. It forms a broad slightly curved oval cell band with a ventromedial to dorsolateral orientation (Fig. 3). Its ventral pole is situated dorsolaterally to the MNTB. Most neurons in the MSO are spindle-shaped or oval, but many triangular and multipolar cells are intermixed. The neurons are arranged in an irregular way, but most spindle-shaped neurons are directed with their long axes running ventromedial to dorsolateral.

The Medial Nucleus of the Trapezoid body. The MNTB is situated just dorsolateral to the pyramidal tract. The cells composing the nucleus are very homogeneous in size and shape. They are round, medium-sized, and have a small, eccentric nucleus. The neurons are arranged in ten to 15 horizontal rows between the crossing fibers of the ventral trapezoid body.

The Nuclei of the Lateral Lemniscus. Between the SOC and the inferior colliculus there are three nuclei which project to the IC as the HRP experiments have shown. These nuclei are surrounded by the ascending and descending fibers of the lateral lemniscus. They are called the ventral (VNLL), intermediate (INLL), and dorsal (DNLL) nuclei of the lateral lemniscus (Fig. 4).

The Ventral Nucleus of the Lateral Lemniscus. The VNLL can be divided on the basis of cell-stained material into a lateral and a medial part. The medial part appears very homogeneous and consists of densely packed, medium-sized, spherical cells. Caudally the 

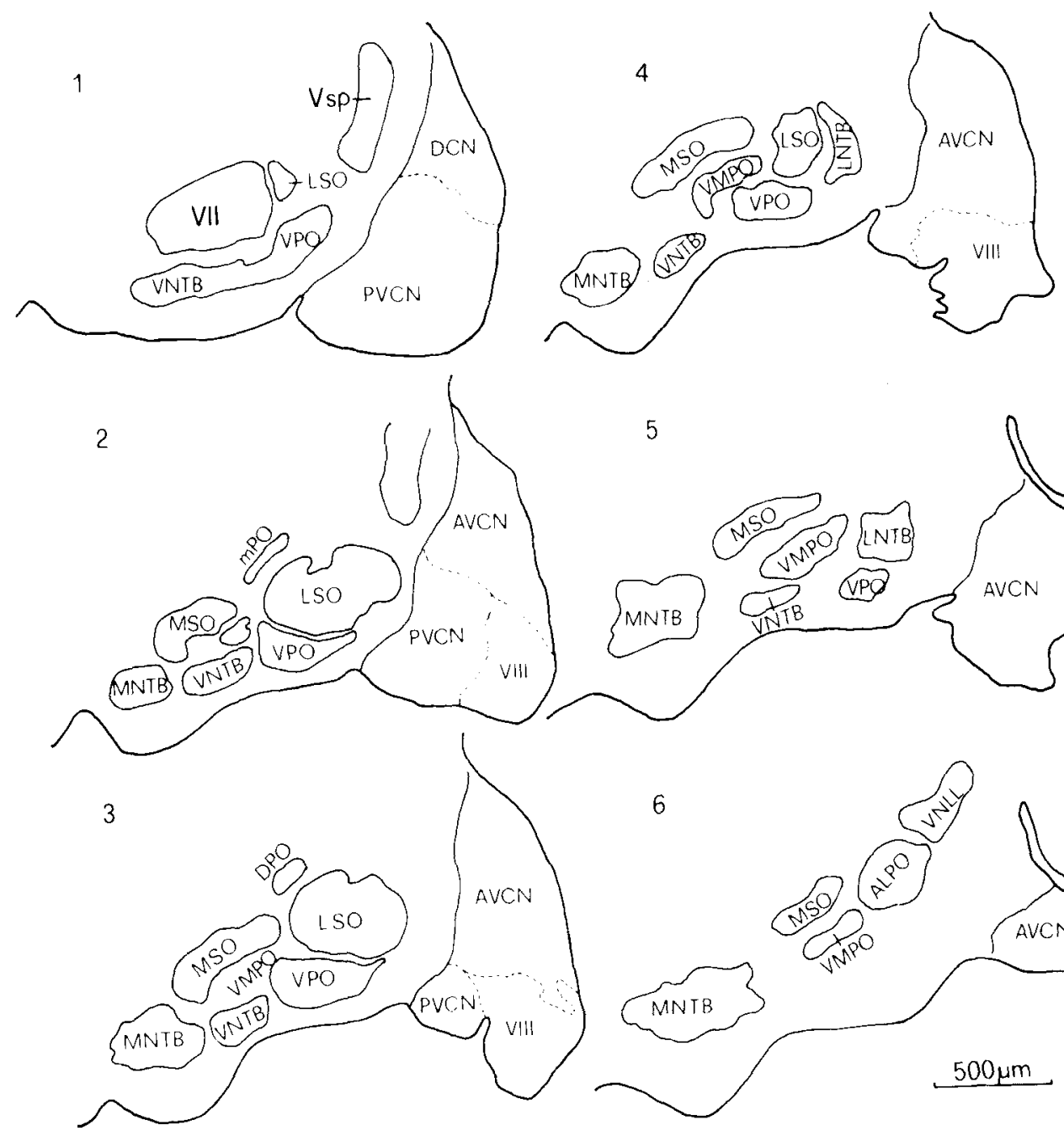

5

Fig. 3. Frontal sections of the superior olivary complex. Section 1 is most caudal, section 6 most rostral. The sections are spaced at $180 \mu \mathrm{m}$. Abbrev.: $\mathrm{mPO}-$ marginal periolivary

cell group; $\mathrm{V} \mathrm{sp-nucleus} \mathrm{of} \mathrm{the} \mathrm{spinal} \mathrm{tract} \mathrm{of} \mathrm{the} \mathrm{trigeminal}$ nerve; VII-motor nucleus of the facial nerve; VIII-acoustic nerve root.

cells are arranged in vertical rows between the lemniscal fibers. In the lateral part the cells are much more loosely packed and many large multipolar, globular, and elongate cells are found in addition to the spherical cells. The region between the two parts shows a very low cell density. The dorsal border of the VNLL is defined by lemniscal fibers crossing from the lateral side at a right angle to the main pathway to the medial side and/or vice versa.

The Intermediate Nucleus of the Lateral Lemniscus. The INLL has about the same size as the VNLL, is continuous with it dor-

sally, and forms a lateral bulge in the surface of the brainstem. The nucleus mainly consists of fusiform cells which are arranged with their long axes running in a lateral to medial orientation. Many fiber bundles of the lateral lemniscus cross through the nucleus like the bundle at the dorsal pole of the VNLL.

The Dorsal Nucleus of the Lateral Lemniscus. The DNLL is located dorsally to the INLL just ventral to the external nucleus of the inferior colliculus. It consists of different cell types which have long dendrites often ex- 

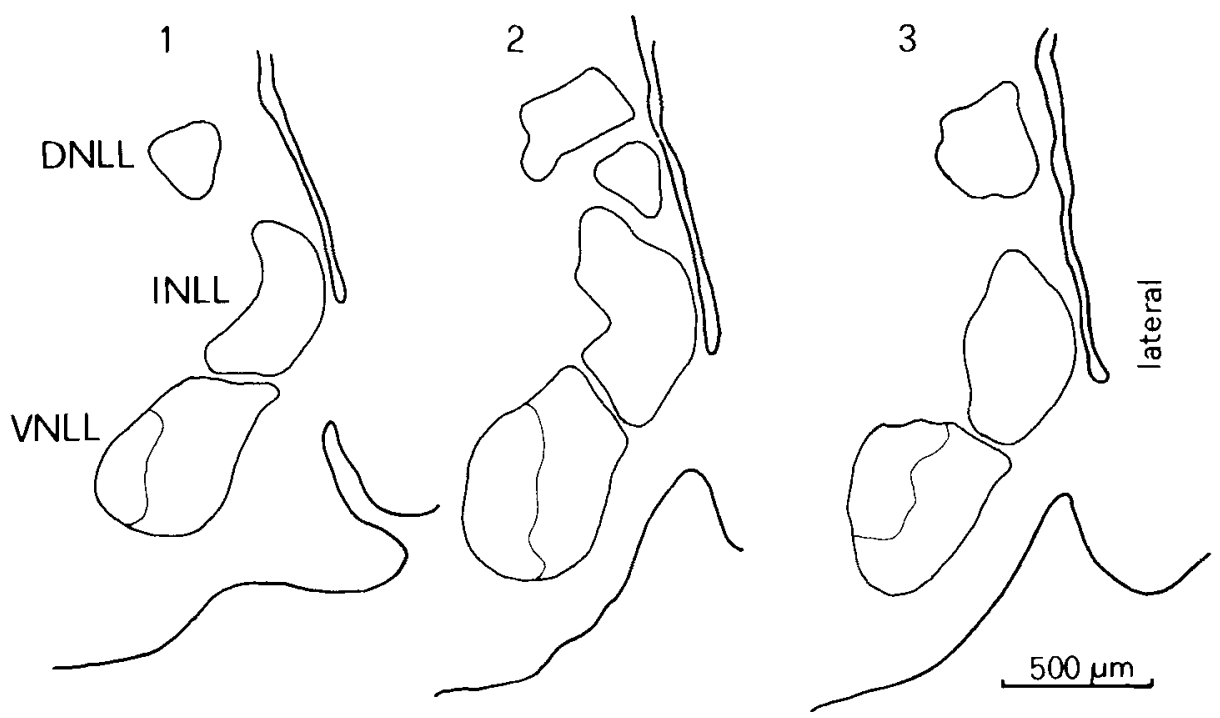

Fig. 4. Frontal sections of the nuclei of the lateral lemniscus. Section 1 is caudal, section 3 rostral. The sections are spaced at $180 \mu \mathrm{m}$.

tending dorsally into the ventral parts of the IC.

The Inferior Colliculus. As in most other microchiroptera, the IC of Rhinolophus ferrumequinum can be seen directly at the brain surface after removal of the dorsal parts of the skull. Only its most caudal parts are covered by the cerebellar vermis. The IC is bordered ventrally by the superior cerebellar peduncle and the parapeduncular nucleus and more rostrally by the DNLL, the cuneiform nucleus, and the incoming fibers of the lateral lemnicus. The rostral border is formed by the deep layers of the superior colliculus and the intercollicular area. Medially the IC is bordered by the central gray matter.

The IC of Rhinolophus can be divided into four parts (Fig. 5). These parts are named according to their spatial relationships: central nucleus (ICC), pericentral nucleus (ICP), posterior nucleus, and external nucleus (ICX). All these nuclei are cytoarchitecturally different and seem to have different connections.

The Central Nucleus of the IC. The central nucleus is the main nucleus of the IC. It occupies by far the largest portion of the posterior midbrain tectum. Within the ICC it is possible to distinguish a dorsolateral, a ventromedial, and a dorsomedial region. Although the cytoarchitecture is clearly different there exist only gradual transitions between adjacent regions. Furthermore, most of the cell types can be found in all three regions, but the relative percentage of neuron types is quite different. The most impressive difference, however, is the density of cells and the distribution of large cells.

On the basis of cell-stained material, two main classes of neurons can be distinguished in the ICC: (1) multipolar cells, which appear round, oval, elongate, or polygonal, with a broad range of soma diameters (from about 15 to $35 \mu \mathrm{m}$ ), and (2) fusiform cells, which appear elongate or ovoid.

The Dorsolateral Region of the ICC. The neurons in the dorsolateral region are much more densely packed than in the ventromedial region. The fusiform neurons are arranged in layers, turning from dorsomedial to ventrolateral, and they run parallel to the ascending lateral lemniscus fibers. Medium-sized and a few large, multipolar cells are intermixed with the fusiform cells, especially dorsally and dorsolaterally at the border to the ICP. The dorsolateral region is only $500 \mu \mathrm{m}$ thick medially, but extends laterally to a depth of about 1,800 $\mu \mathrm{m}$.

The Ventromedial Region of the ICC. In contrast to the dorsolateral region, the ventro- 

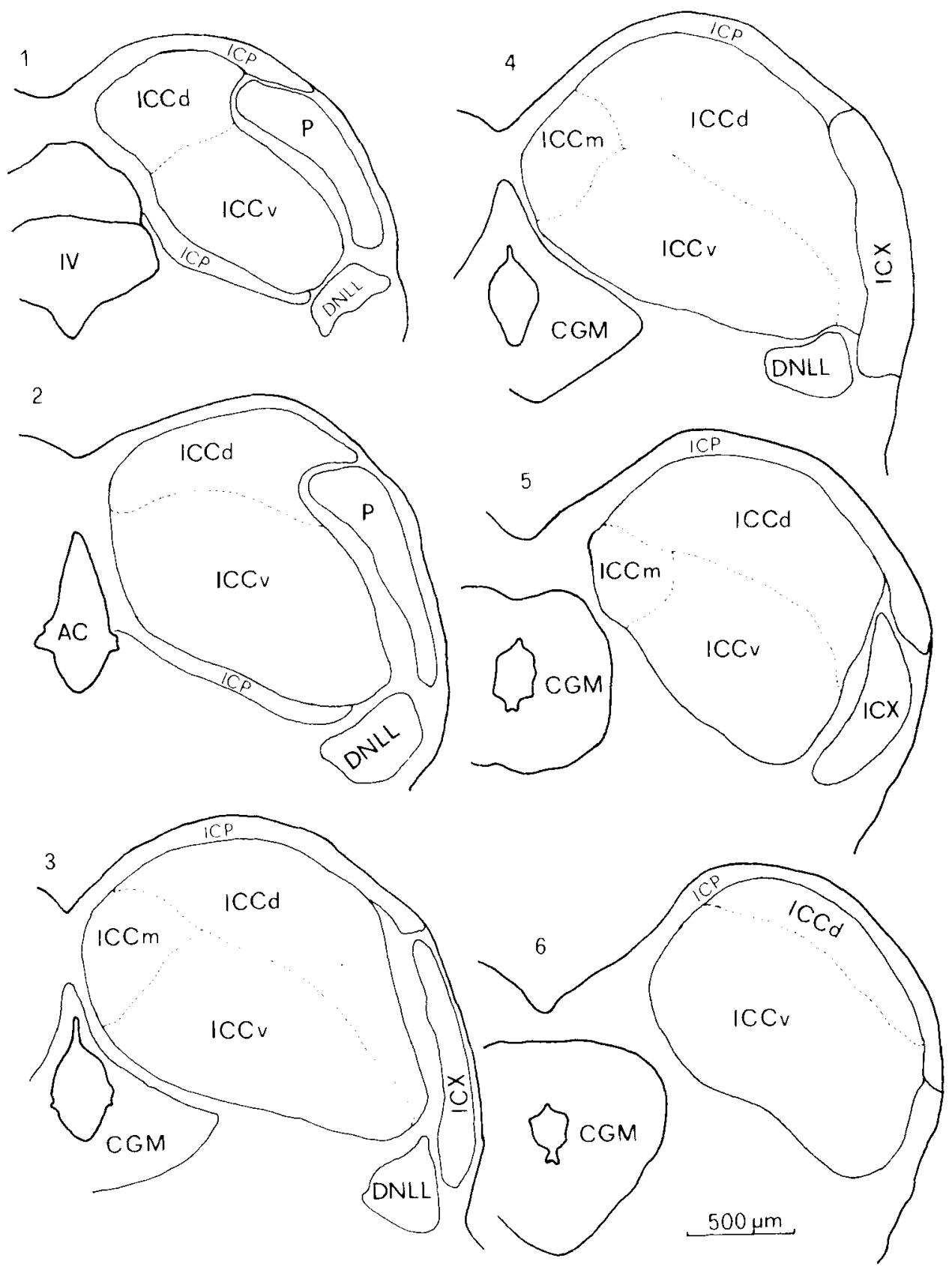

Fig. 5. Frontal sections of the inferior colliculus. Section 1 is caudal, section 6 rostral. The sections are spaced at 180 $\mu \mathrm{m}$. Abbrev.: AC-cerebral aquaeduct; ICCd-dorsolateral, ICCm-dorsomedial, ICCv-ventromedial region of the ICC; $\mathrm{P}$-posterior nucleus; IV-fourth ventricle. 
medial region does not show any stratification in the cell arrangement and the neurons are not densely packed. More multipolar and other large cells are intermixed with the fusiform cells.

The large cells show a typical arrangement in the ICC. In the caudal third they are congregated ventrolaterally and ventromedially. In the middle parts of the ICC only a few large cells can be observed. In the rostral third the number of small cells and the cell density decrease and this part of the ICC characteristically has a large number of medium-sized and large neurons. The ringlike distribution of large cells around the ICC can be seen most clearly in sagittal sections. They are accumulated all around the ICC, except in its dorsocaudal part, where they are only sparsely distributed.

The Dorsomedial Region of the ICC. The characteristic feature of the dorsomedial region is a high number of small multipolar cells; these, in addition to small fusiform cells, give a granular appearance to this region.

All these data based on cell-stained material clearly show that the ICC is not homogeneous in its structure but can be divided into different parts which might receive different connections from the lower and higher auditory centers and might have different functions in auditory processing.

The Pericentral Nucleus of the IC. The pericentral nucleus in not very well developed in Rhinolophus and it is sometimes difficult to separate it from the dorsomedial and dorsolateral region of the ICC. It covers the surface of the ICC as a thin shell ( 100 to $200 \mu \mathrm{m}$ thick) caudally, medially, dorsally, and rostrally. It can mainly be distinguished from the ICC by its lower cell density. Most cells are spindleshaped and are directed with their long axes parallel to the brain surface.

The Posterior Nucleus. This nucleus, which seems to have no homologue in other mammals, is situated caudally in the lateral portion of the IC (Fig. 5). It is separated from the ICC by a region of very low cell density. The nucleus is homogeneous in appearance and is mainly composed of small, round cells which are densely packed.

The External Nucleus of the IC. The rostral continuation of the posterior nucleus forms the external nucleus containing the fibers coursing to the brachium of the IC. It is composed of loosely packed large cells of different shape. In the caudal parts of the ICX, at the border to the ICC, very large cells occur. These are the largest cells in the whole IC (40- to 50- $\mu \mathrm{m}$ soma diameter) and they seem to connect the ICC with the ICX. The neurons are directed from dorsolateral to ventromedial and their long dendrites (up to $400 \mu \mathrm{m}$ ) cross laterally through the ICX and lead medially deep into the ICC.

Fiber Architecture of the IC. In fiberstained material five fiber bundles can be seen coursing into or out of the IC, and they show a characteristic course and distribution within the IC. These fiber bundels are the lateral lemniscus (LL), the commissure of the IC (CIC), the commissure of Probst, the brachium of the IC (BIC), and the tectopontine tract.

The lateral lemniscus represents the main auditory input to the IC from lower auditory nuclei. It contains fibers from and to $\mathrm{CN}$, SOC, and the nuclei of the lateral lemniscus. Before reaching the IC the fibers of the LL cross as compact bundles through the region of the DNLL. The LL fibers enter the IC ventrally, but ventrolaterally and ventromedially they are combined into two main bundles. The main direction of the LL fibers within the IC is from ventral to dorsomedial.

A strong connection exists between the two IC. Fibers having their somata in the ICP and the ICC of both sides take a ventral to dorsomedial pathway within the IC and cross in the commissure of the IC. The course of the commissural fibers within the IC is nearly parallel to the ascending lemniscal fibers.

The fibers of the commissure of Probst enter the IC ventromedially intermixed with $L L$ fibers. As the HRP experiments will show, the fibers of the commissure of Probst are axons of the contralateral DNLL neurons crossing the midline at the bottom of the central gray matter, just dorsal and ventral to the medial longitudinal fascicle. Their course within the IC seems to be parallel to the LL fibers.

The fibers of the brachium of the IC and of the tectopontine tract take a totally different path within the IC with respect to the LL fibers. In general they run from medial to lateral, nearly at a right angle to the ascending lemniscal fibers. At the border to the ICX they turn ventrally and form the BIC at the lateral edge of the IC and just ventral to it is the tectopontine tract.

In summary, the fiber pattern of the IC is formed by incoming LL fibers running from 
ventral to dorsal and crossing at nearly a right angle the medially to laterally directed outgoing fibers of the BIC and of the tectopontine tract.

\section{Part II: The connections of the inferior colliculus}

The results on the connections of the IC are based on six representative experiments in which HRP was injected into different parts of the IC. Because of the relatively large HRP injections and the overrepresentation of the ICC compared to ICP and ICX, this study can only provide information on the afferent projections to the entire IC and only a limited amount of information on the terminations of the projecting fibers in different subdivisions of the IC.

After HRP injections into the IC, labeled cells were found in the following nuclei: ipsilaterally in the IC, the DNLL, the VNLL, the INLL, the MSO, the LSO, the VNTB, the VPO, the VMPO, the LNTB, the ALPO, the DPO, the $D C N$, the auditory cortex, the suprapeduncular nucleus, the nucleus of the BIC, the cuneate nucleus, the gracile nucleus, the nucleus of the spinal tract of the trigeminal nerve, and the raphe nucleus; contralaterally labeled neurons were found in the IC, the DNLL, the LSO, the VNTB, the VPO, the VMPO, the DCN, the PVCN, the AVCN, the cuneate nucleus, the gracile nucleus, and the nucleus of the spinal tract of the trigeminal nerve.

Anterograde transport of HRP and terminal axon arborization were evident ipsilaterally in the medial geniculate body (MGB), the lateral pontine nuclei, the deep layers of the superior colliculus, the central gray matter adjacent to the IC, scattered in the pontine reticular formation, and contralaterally in the IC. Axon terminal arborization also was found ipsilaterally in the VNLL, the MSO, the LSO, the VNTB, and contralaterally in the LSO, but these projections must be confirmed by other, anterograde methods.

In the following the position of the injection sites in the IC and the distribution of labeled cells will be discribed in three representative experiments where HRP was injected into the whole IC (one animal, experiment RF17), the deep parts of the ICC (experiment RF12), and into the dorsolateral region of the ICC, including parts of the ICP (experiment RF18). The results of the other experiments are very similar and are included in a summarized presentation when they differ from that of three described cases.
In the experiment where a large HRP deposit was placed into the caudal portions of the IC (RF17; Fig. 6), the entire caudal half of the IC was filled with HRP reaction product including ICP, the posterior nucleus, and parts of the ICX. In the rostral half the affected region was restricted to an elongated band reaching from dorsomedial to ventrolateral through the ICC.

The region of labeled cells in the contralateral IC corresponded to the injected IC portion but was smaller than the dark brown injection zone. Because of the high HRP concentration most labeled neurons in the ICC were completely stained as in a Golgi preparation (Fig. 7A). No labeled cells were found in the contralateral ICX and only a few in the ICP.

In the nuclei of the lateral lemniscus labeled cells were found contralaterally in the DNLL and ipsilaterally in the DNLL, the INLL, and the VNLL. In the DNLL and INLL the labeled cells were scattered throughout the nuclei. Labeling in the VNLL was concentrated in the lateral half of the nucleus. In the medial half only a few cells were labeled in the most ventromedial region.

In the SOC, labeled neurons were found contralaterally in the LSO, VNTB, and VPO, and they were found ipsilaterally in the LSO, MSO, VNTB, VPO, VMPO, and a few in the LNTB, ALPO, and DPO. The labeled neurons in the MSO were distributed throughout the nucleus. In the LSO they were found ispi- and contralaterally in all parts of the nuclei, but the cells located in the medial part of each nucleus were much more heavily labeled than those found in other parts of the LSO.

All three contralateral nuclei of the cochlear nucleus complex showed heavy labeling. Ipsilaterally labeled cells were found only in the dorsocaudal region of the DCN. In the contralateral DCN labeled cells were found in the dorsocaudal region and in the fusiform region of the remaining nucleus. The labeled neurons in the contralateral PVCN were situated in the ventral, the lateral, and the central subdivisions (Fig. 9A). The AVCN contained labeled cells throughout the nucleus, but the density of labeling was higher in caudal parts (Fig. 9A). Only the most rostral parts of the AVCN were completely unlabeled.

In addition to cells labeled in the lower auditory centers, HRP was taken up by the large pyramidal cells of layer $\mathrm{V}$ of the auditory cortex (Fig. 7B). Furthermore, some labeled cells were located in the thalamus medial to the BIC and dorsolateral to the cerebral peduncle. At the level of the LSO some neurons were labeled 

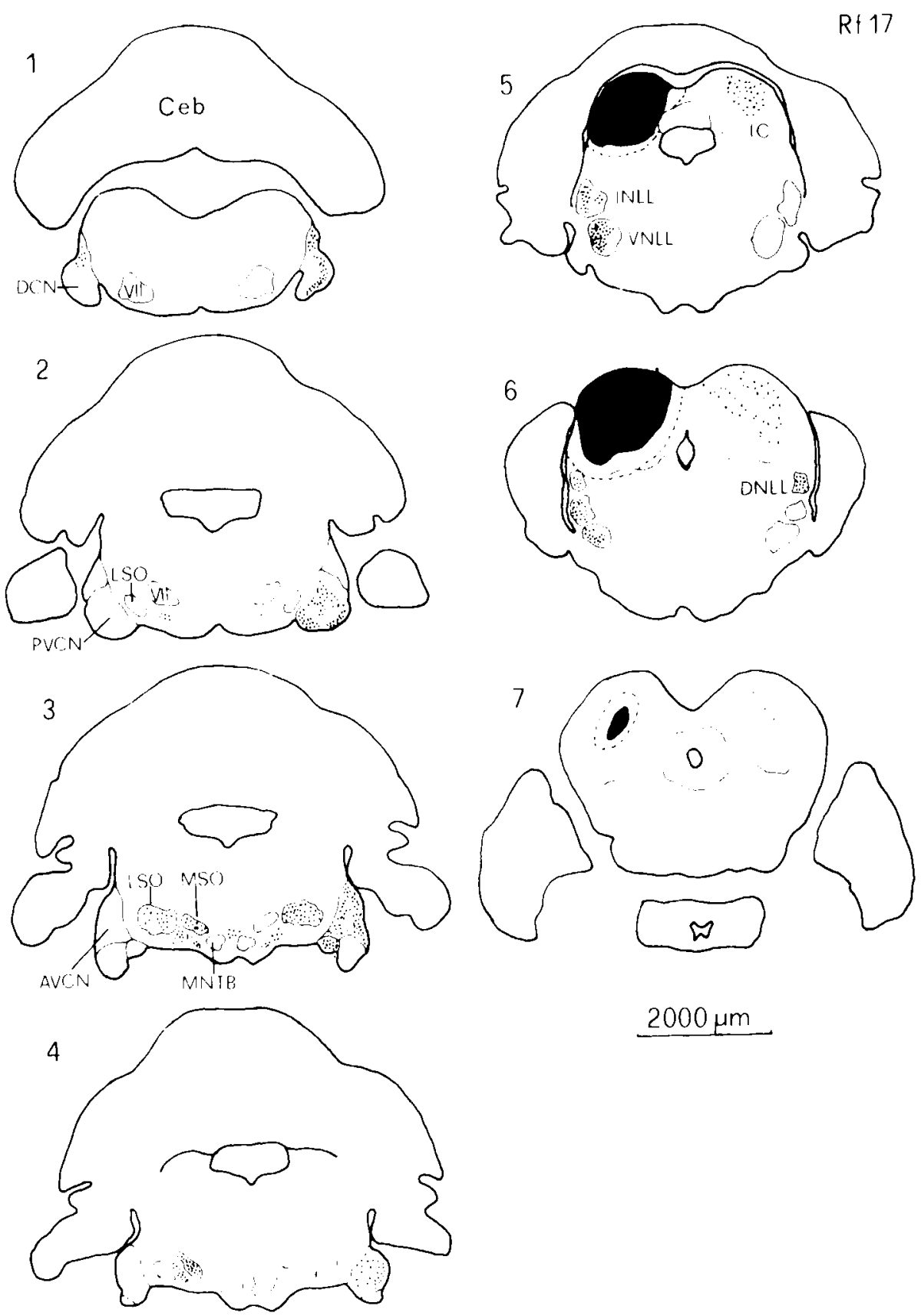

\section{$2000 \mu \mathrm{m}$}

Fig. 6. Frontal sections of the brainstem showing the distribution of HRP-labeled neurons in the lower auditory nuclei in the experiment RF17. Section 1 is most caudal, section 7 most rostral. The sections are spaced at $400 \mu \mathrm{m}$.

The black area in the IC surrounded by a dotted line indicates the position of the HRP deposit. Abbrev:: VII-motor nucleus of the facial nerve. 

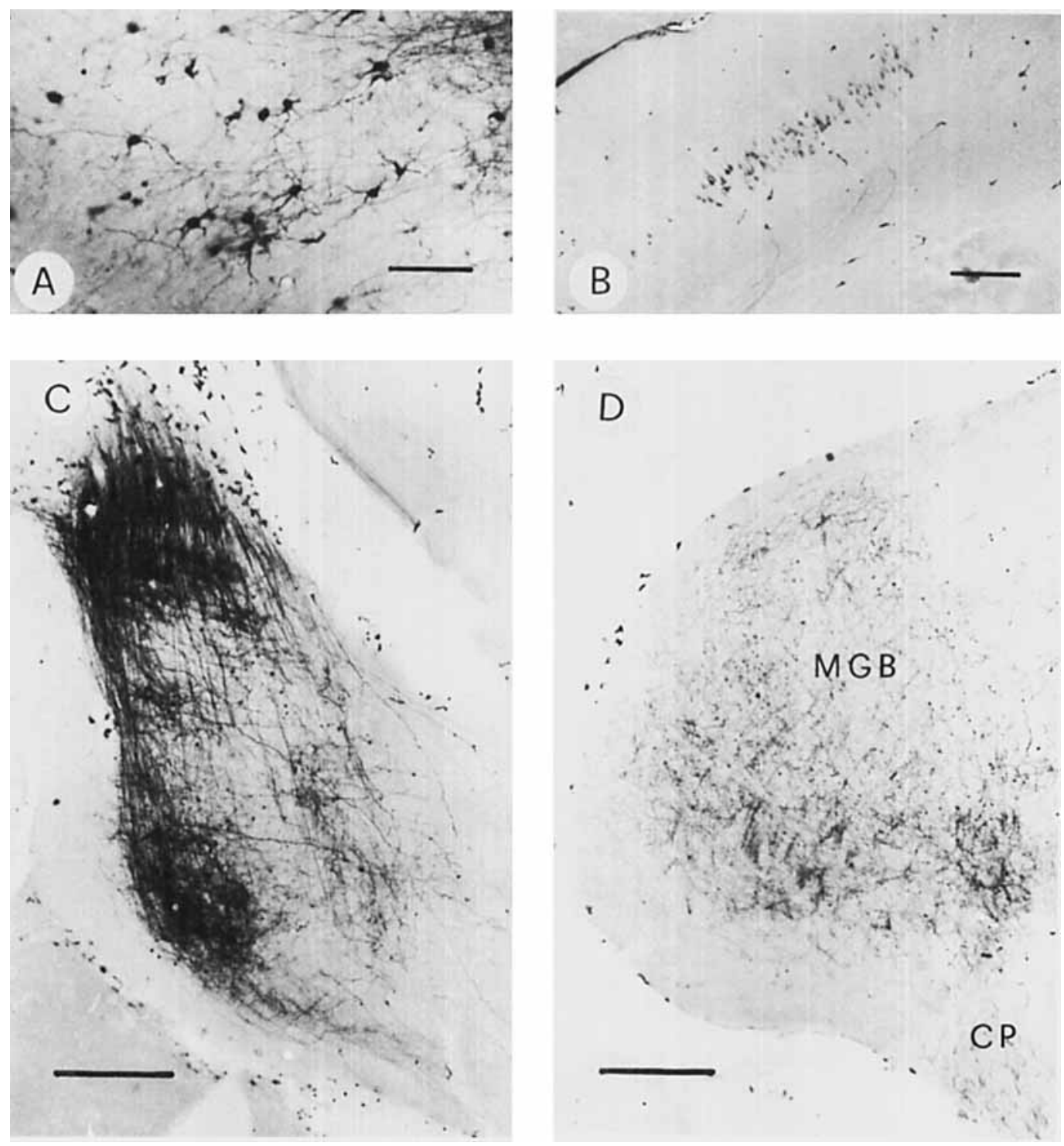

Fig. 7. A: Golgi-like labeled neurons in the contralateral ICC. Note that most neurons are multipolar. Calibration bar $100 \mu \mathrm{m}$. B: Labeled pyramidal cells in layer $V$ of the ipsilateral auditory cortex. Calibration bar $200 \mu \mathrm{m}$. C: An-

terograde labeling within the ipsilateral pontine nuclei. Calibration bar $200 \mu \mathrm{m}$. D. Anterograde labeling in the ipsilateral MGB and labeled fibers in the cerebral peduncle (CP). Calibration bar $200 \mu \mathrm{m}$.

in the raphe nucleus between the two MNTBs. Labeled cells were also found ipsi- and contralaterally in the cuneate nucleus, in the gracile nucleus, and in the nucleus of the spinal tract of the trigeminal nerve.

Anterograde labeled axons and axonal arborization occurred ispilaterally in the medial

geniculate body (Fig. 7D) and in the ipsilateral pontine nuclei (Fig. 7C).

Figure 8 shows the injection site in the IC and the distribution of labeled cells in the brainstem in an experiment (RF 12) where HRP was injected into middle and deep parts of the ICC. Only small parts of the ICP and no 

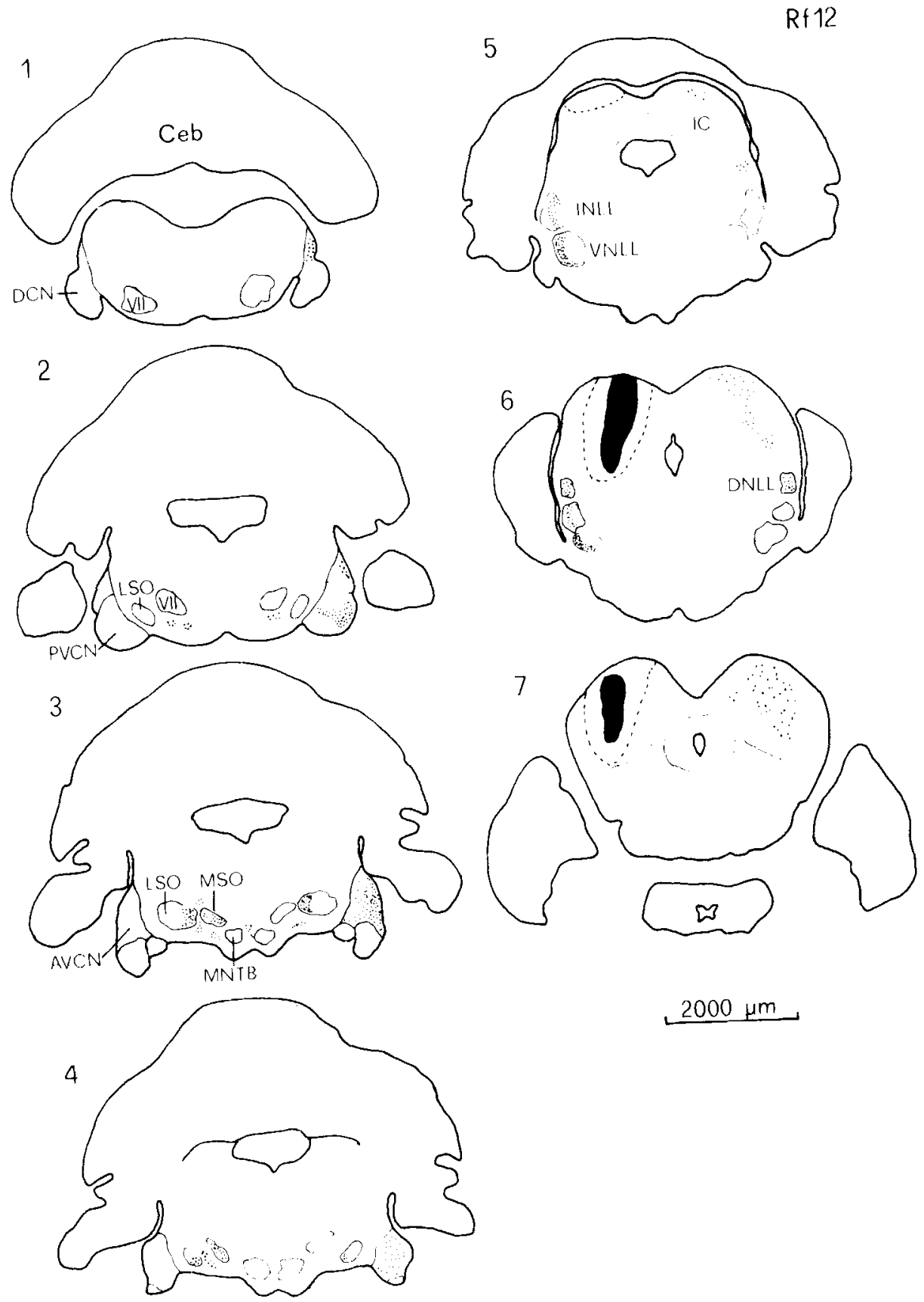

Fig. 8. Frontal sections of the brainstem showing the distribution of labeled cells after a deep IC injection (RF12). Section 1 is most caudal, section 7 most rostral. The sections are spaced at $400 \mu \mathrm{m}$. The black area indicates the position of the HRP deposit. Note the topographical arrangment of labeled cells in different nuclei. Abbrev: VII-motor nucleus of the facial nerve. 

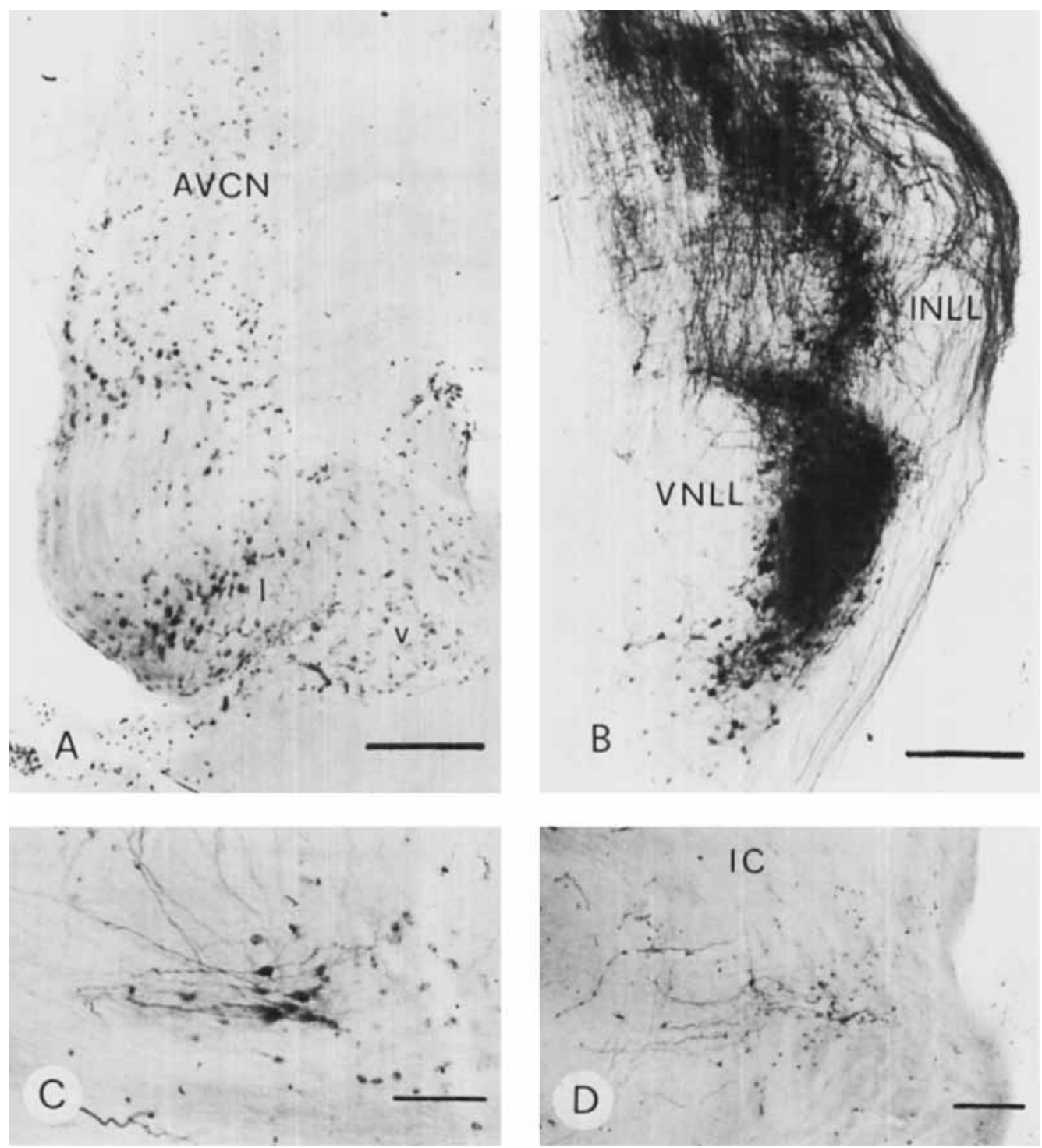

Fig. 9. A: Labeled neurons in the contralateral cochlear nucleus. In the PVCN, cells are labeled in the ventral (v) as well as in the lateral (1) subdivision. Calibration bar $200 \mu \mathrm{m}$. B: Labeling pattern in the ipsilateral nuclei of the lateral lemniscus. Calibration bar $200 \mu \mathrm{m}$. C: Labeled bipolar cells in the VNTB. Calibration bar $100 \mu \mathrm{m}$. D: Labeled neurons in the contralateral DNLI. Calibration bar $200 \mu \mathrm{m}$.

parts of the posterior nucleus or of the ICX were affected by the injection.

Labeled cells in the contralateral ICC were found in locations corresponding to the ipsilateral injection site.

Heavy labeling occurred in all three nuclei of the lateral lemniscus ipsilaterally, whereas contralaterally only the DNLL contained la- beled neurons. Labeled axons could be traced from the contralateral DNLL neurons up to the injected region in the IC (Fig. 9D). The labeled cells in the ipsilateral INLL and VNLL were concentrated in the lateral half of each nucleus, where they form a dorsoventral band (Fig. 9B). Whereas in the INLL many unlabeled cells were intermixed with labeled neu- 
rons, in the lateral half of the VNLL nearly every neuron contained HRP reaction product.

In the superior olivary complex labeled neurons were found ipsilaterally in tbe LSO, MSO, VNTB, VPO, DPO, and ALPO and contralaterally in the LSO, VNTB, and VMPO. In caudal sections, the first labeled neurons in the SOC appeared in the VNTB ventromedial to the motor nucleus of the facial nerve. Most neurons were bipolar with long dendrites running parallel to the trapezoid body fibers (Fig. 9C). Similar neurons were found in the VPO. Many cells were labeled in the VMPO especially in the rostral parts of the SOC where the labeled neurons formed a cellular band passing from ventromedial to dorsolateral between the LSO and the MSO. A few multipolar cells containing HRP reaction product were present just dorsal to the space between the MSO and the LSO in the DPO. Most labeled cells in the LSO of both sides were fusiform and situated only in the medial curvature of the nuclei (Fig. 10A). Only a few labeled cells were observed in the more ventrolateral parts. Labeling in the MSO occurred ipsilaterally in the ventromedial half of the nucleus in caudal sections, whereas in more rostral sections labeled cells were scattered throughout the nucleus (Fig. 10B).

Heavy labeling was found contralaterally in all three subnuclei of the cochlear nucleus complex. The labeled neurons in the DCN were concentrated in the dorsocaudal region; only a few labeled cells were found in the fusiform cell region throughout the nucleus and in the polymorphic cell region. In the PVCN labeled cells were concentrated in the lateral subdivision. No labeled cells were situated in the ventral subdivision and only a few occurred in the central subdivision. In the caudal third of the AVCN the labeled neurons were concentrated laterally, distributed throughout the nucleus in middle parts, and only poorly represented in the anterior third. The most rostral portion of the AVCN was not labeled.

In addition to labeling in brainstem auditory nuclei, some labeled neurons were found in the raphe nucleus between the two MNTBs and in the reticular formation at the level of the motor nucleus of the facial nerve. In the diencephalon labeled cells were found just medial to the BIC and in a distinct cell group just dorsolateral to the lateral parts of the cerebral peduncle. Labeled axons could be detected in the cerebral peduncle and these probably arose from the auditory cortex. Heavy anterograde labeling was found in the ipsilateral MGB and the lat- eral pontine nuclei. Some labeled axons could be followed into the central gray matter of both sides and into the deep layers of the superior colliculus of the same side.

In a further experiment (RF 18), the injection site was situated dorsolaterally in the IC (Fig. 11). It covered dorsolateral parts of the ICP and parts of the dorsolateral region of the ICC: the ventromedial and the dorsomedial region of the ICC and the ICX were spared.

Many labeled cells were found in the ipsilateral dorsomedial region of the ICC. A few labeled cells were present dorsally and dorsolaterally in the contralateral ICC. The labeled cells appeared in about the same region and at the same depth as the electrode tip was positioned in the ipsilateral IC during iontophoretic injection.

In the nuclei of the lateral lemniscus labeled cells were found ipsilaterally in the DNLL, INLL, and VNLL, and contralaterally only in the DNLL. While in the DNLL of both sides labeled neurons were scattered throughout the nuclei, in the INLL and especially in the VNLL a clear topographical arrangement of labeled neurons could be observed. In the INLL the labeled cells were loosely arranged in dorsal and dorsolateral parts of the nucleus. In the VNLL labeled cells were located medially from the dorsal to the ventral border of the nucleus.

Labeling in the SOC was rather faint. Most labeled cells appeared ipsilaterally in the VMPO. In the LSO the labeled neurons were located ipsi- and contralaterally in the most lateral parts of the nuclei. Only a couple of faintly labeled neurons were present dorsally in the ipsilateral MSO. In addition some labeled cells were observed ipsilaterally in the VNTB, VPO, LNTB, and ALPO.

In contrast to the SOC the contralateral cochlear nucleus showed heavy labeling. Many cells were labeled in the fusiform cell layer and quite a few in the polymorphous cell region of the DNC, whereas the dorsocaudal region of the DCN was free of labeling. A couple of cells were also labeled in the fusiform cell layer of the ipsilateral DCN. In the PVCN labeled cells were were located exclusively in the ventral subdivision, extending throughout the caudorostral extent. In the AVCN labeled cells were found primarily in the lateral portions of the rostral third part.

Some labeled pyramidal cells were found in layer V of the ipsilateral auditory cortex. Similar to all other cases, anterograde labeled axons occurred in the MGB and the lateral pontine nuclei. Many labeled axons could be 



Fig. 10. A: Labeling pattern within the ipsi- (on the right side) and contralateral superior olivary complex after a ventral IC injection. Note that no cells are labeled in the contralateral MSO. Calibration bar $500 \mu \mathrm{m}$. B: Labeling in the ipsilateral superior olivary complex. (The same section as in Fig. 10A, at higher magnification), Calibration bar 250 $\mu \mathrm{m}$. 

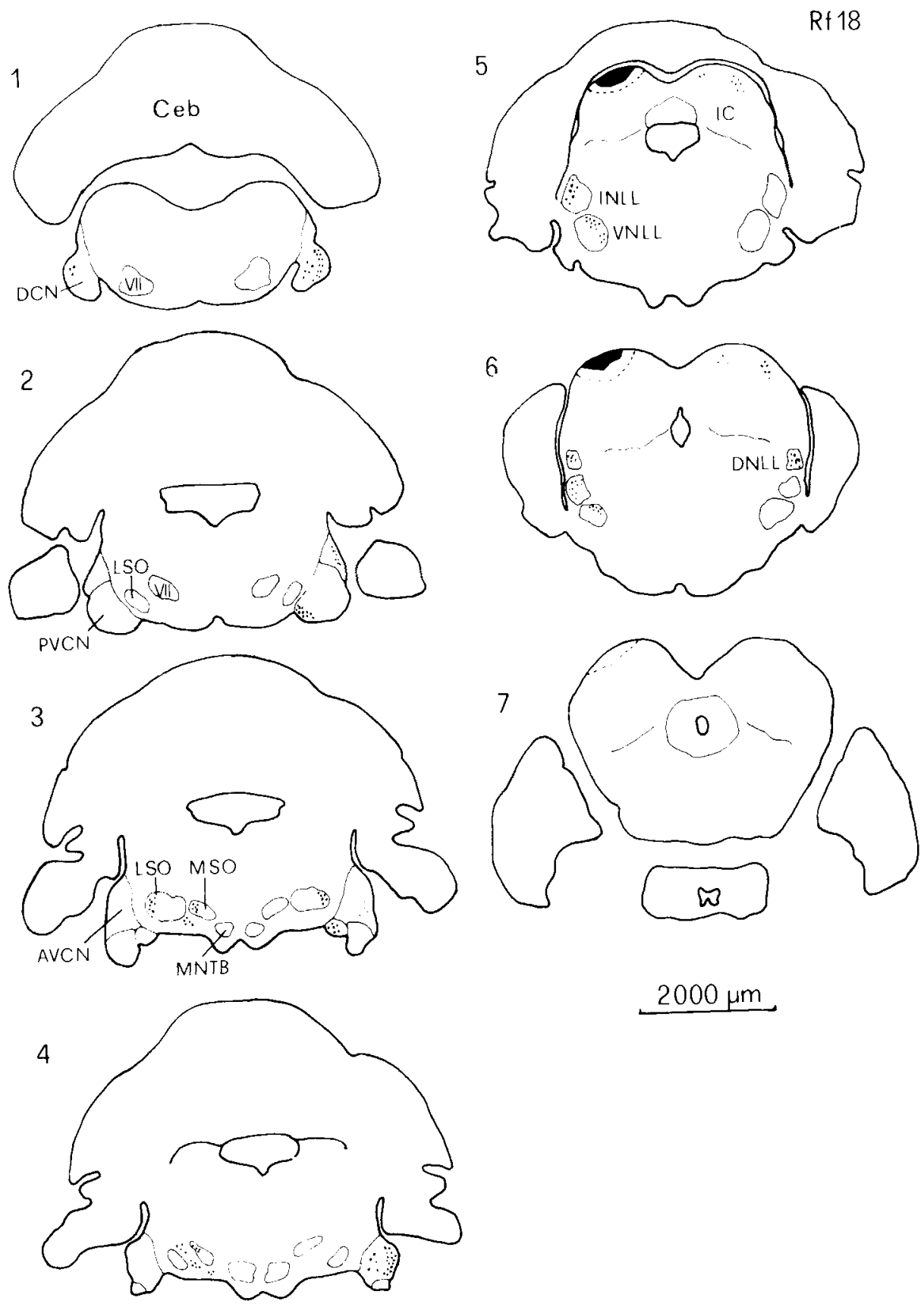

Fig. 11. Frontal sections of the brainstem showing the distribution of labeled neurons in the lower auditory nuclei in the case of a dorsal IC injection (RF18). Section 1 is most caudal, section 7 most rostral. The sections are spaced at

$400 \mu \mathrm{m}$. The black area indicates the position of the HRP deposit in the IC. Note the difference projection pattern in comparison to RF12 (Fgi. 8). Abbrev.: VII-motor nucleus of the facial nerve. 
followed from the injection site to the central gray of both sides, but their terminal fields could not be determined.

The results of all HRP experiments can be summarized as follows. Relatively large HRP deposits were placed in the IC. Two main cases can be distinguished: (1) The HRP deposit was located in the dorsal parts of the IC including the ICP, the dorsal parts of the ICC, and dorsal parts of the ICX; (2) the HRP deposit was placed deep into the ICC and the injection site included parts of the ICP, the dorsolateral, and the ventromedial region of the ICC. In most experiments the dorsomedial region of the ICC and the ICX were missed. Also spared were the most anterior parts of the ICC to avoid spreading of HRP into the superior colliculus.

After the HRP reaction labeled neurons and/ or labeled axon terminals were found in different brainstem nuclei and in layer $\mathrm{V}$ of the auditory cortex. While the overall projection pattern was similar in all cases, some auditory nuclei showed different labeling patterns depending on the injection site in the IC.

In all experiments labeled cells were found in the IC contralateral to the injection site and axons could be tracked in the commissure of the IC. Only a few labeled cells were found in the contralateral ICP and none in the posterior or the external nucleus. Most cells in the contralateral ICC seemed to be small and medium-sized multipolar cells, as judged from Golgi-like labeling.

In all cases labeled cells could be found in all three nuclei of the lateral lemniscus. However, whereas the DNLL projected bilaterally, the INLL and VNLL projected only ipsilaterally. No topographic representation of labeled cells could be observed in the DNLL. In the INLL, labeled cells were sometimes concentrated in more medial parts and sometimes in more lateral parts, but the regions where labeled cells were found were not consistently related to the position of the injection site. In contrast to the DNLL and the INLL the position of labeled cells in the VNLL was clearly related to the injection site. Labeled cells were localized in medial parts in the case of dorsal IC injections and were concentrated in a lateral band in the case of deep IC injections.

In all experiments the projection pattern of SOC neurons was very similar. Labeled cells were detected ipsilaterally in the LSO, MSO, VNTB, VPO, VMPO, DPRO, LNTB, and ALPO and contralaterally in the LSO, VNTB, and the VPO. In no case were labeled cells found in the medial nucleus of the trapezoid body. With dorsal IC injections labeled cells were found more dorsally in the MSO and laterally in the LSO and with deep IC injections ventromedially in the MSO and medially in the LSO. No topographical arrangement of labeled cells could be found in the periolivary nuclei.

The three subnuclei of the cochlear nucleus complex mainly projected contralaterally to the IC. Only a few labeled cells were found in the ipsilateral DCN. In the contralateral CN distinct topographical representation occurred in relation to the position of the HRP deposit. In the case of dorsal IC injections labeled neurons were located ventrally in the PVCN and the DCN and rostrally in the AVCN. In the DCN most labeled cells were located in the fusiform cell layer. In the case of deep IC injections, labeled cells were found predominantly in the lateral subdivision of the PVCN, in the dorsocaudal region of the DCN, and posteriorly in the AVCN. At the diencephalic level labeled cells were found just medial to the BIC in the nucleus of the brachium of the IC and a second cell group was located ventromedially to the MGB in the suprapeduncular nucleus. No labeled cells were found in the MGB itself, but a very dense network of labeled axons and axonal arborization was observed.

In all cases in which the forebrain was sectioned, labeled pyramidal cells were found in layer $\mathrm{V}$ of the temporal cortex. This area corresponds to the auditory cortex. Axons arising from pyramidal cells could be followed through the internal capsule and the cerebral peduncle descending to the IC.

In addition labeled cells were found in nuclei not directly correlated to the classical auditory pathways. In cases where the ICX was covered by the HRP deposit, labeled cells were found bilaterally but mainly contralaterally in the cuneate nucleus, in the gracile nucleus, and in the caudal part of the nucleus of the spinal tract of the trigeminal nerve. Single labeled cells were found scattered in the reticular formation, but they were concentrated in the raphe nucleus, between the two MNTB.

In every case a heavy anterograde transport of HRP occurred and labeled axon terminals could be detected in the lateral pontine nuclei and the medial geniculate body. Some labeled axons seemed to terminate in the pontine reticular formation, in the central gray matter, and in the deep layers of the superior colliculus.

\section{DISCUSSION}

Since the work of Poljak $(26 a, b)$ and other (for review see Henson, '70) there have been 
only a few anatomical investigations on the central auditory system of microchiroptera (Schweizer, '78; Zook, '79; Schweizer and Radtke, '80; Zook and Casseday, '80). The cytoarchitectonic results presented in this report demonstrate that the anatomy of the central auditory pathways of Rhinolophus ferrumequinum does not differ in general from those in other mammals. However, most auditory centers are very well developed in this bat; this is true for most brainstem centers as well as for the higher auditory centers (the MGB and the auditory cortex) (Schweizer and Ratke, '80).

Three major findings on the anatomical organization of the auditory system of the greater horseshoe bat have to be emphasized and will be discussed in more detail in this paper: (1) Medial to the LSO a cell group exists which seems to be homologous to the MSO in other mammals, judging from the projection pattern to the IC; (2) there is a large nucleus between the VNLL and the DNLL which projects to the IC and which was called the intermediate nucleus of the lateral lemniscus (INLL); and (3) the tonotopical arrangement of the projections to the ICC shows the overrepresentation of the high-frequency regions in the auditory system.

\section{The superior olivary complex}

Of all mammalian auditory centers, the SOC is by far the most complicated, and there is much confusion about its organization and function in different mammals. In the cat, normally three main nuclei are distinguished in the SOC-the lateral superior olivary nucleus (LSO), medial superior olivary nucleus (MSO), and the medial nucleus of the trapezoid body (MNTB). These main nuclei are surrounded by several trapezoid body and periolivary nuclei (e.g., Elverland, '78). Most neurophysiological and anatomical work was performed on these main nuclei and only little is known about the projection pattern and the function of the trapezoid body and periolivary nuclei. The nomenclature of the SOC becomes even more confusing when attempts are made to homologize several nuclei in comparative studies of different mammalian species based only on cytoarchitectural investigations. The SOC in Rhinolophus was divided in this study into LSO, MSO, and MNTB, which are surrounded by the VNTB, VPO, LNTB, DPO, ALPO, and the VMPO. Besides some differences in the nomenclature of periolivary nuclei the main question is whether there exists in microchi- roptera a nucleus which is homologous to the MSO in other mammals. In earlier studies the SOC of the bat Myotis myotis was subdivided into a superior olivary nucleus, a paraolivary nucleus, and a lateral and a medial nucleus of the trapezoid body (Schober, '59). The SOC of Rhinolophus was subdivided into a superior olivary nucleus, an accessory olivary nucleus consisting in its rostral parts of an external and an internal nucleus, a nucleus, of the trapezoid body, and an internal and an external preolivary nucleus (Poljak, '26a).

In a comparative study Irving and Harrison ('67) pointed out that the MSO is very small or absent in the hedgehog, bat, mouse, and mole. Harrison and Feldman '70) and Ollo and Schwartz ('79) found a large superior paraolivary nucleus in the rat and the mouse respectively but only a poorly developed MSO. The criterion for the presence or absence of MSO is that MSO must be composed of bipolar cells with one dendrite directed laterally and one medially. According to this criterion the paraolivary nucleus of microchiroptera and some other mammals cannot be homologous with the MSO because it contains a large number of multipolar cells in addition to the bipolar cells. It has been homologized with the retroolivary nucleus of the cat which gives rise to the olivocochlear bundle. Using phylogenetic criteria however, Brown and Howlett ('72) emphasized that in the rat the superior paraolivary nucleus is homologous to the MSO in the cat.

The presence or absence of the MSO was further correlated with the development of the visual system, the head size (distance between the two ears), and the capacity for high-frequency hearing (Harrison and Irving, '66; Irving and Harrison, '67). The MSO is the first auditory center to receive fibers directly from both sides, whereas the LSO receives direct fibers from the ipsilateral cochlear nucleus only; the contralateral input comes from the cochlear nucleus via the MNTB. The LSO projects bilaterally and the MSO ipsilaterally to the ICC. Neurophysiological data have shown that intensity differences between the two ears are processed in the LSO whereas the MSO decodes time differences (Tsuchitani and Boudreau, '64; Moushegian et al., '72). The LSO is very well developed in microchiroptera; the MNTB which is a direct index for the size of the LSO has the highest progression index of all auditory nuclei in Rhinolophus (Baron, '74), suggesting that processing of intensity differences is a very important cue for echolocation. Most microchiroptera have a poorly developed 
visual system and a small interaural distance, and the frequencies of their echolocation calls and of best hearing are in the ultrasonic range. Therefore one would expect from the hypothesis of Harrison and Irving ('66) that bats have a well-developed LSO and a small or absent MSO, as the encountered interaural time differences are extremely small. But for two reasons the arguments of Brown and Howlett (72), to homologize the nucleus medial to the LSO with the MSO of other mammals, were adopted even though the cytoarchitecture of this region is quite different. First, Harrison and Irving ('66) homologized the paraolivary nucleus with the retroolivary nucleus, the source of the olivocochlear bundle. If this holds, the neurons of this nucleus should be AChEpositive, which is not true in Rhinolophus for the paraolivary nucleus (MSO) (personal observations). Neurons which are AChE-positive were only located within the SOC in the VPO and VMPO, and the olivocochlear bundle is poorly developed. The AChE results support the hypothesis of Brown and Howlett ('72) in the rat. The second reason is the projection pattern to the ICC. The retroolivary nucleus in the cat projects bilaterally to the ICC, whereas the paraolivary nucleus (MSO) of Rhinolophus projects ipsilaterally only. Although the cell composition and cell arrangement in the paraolivary nucleus are different from the MSO in the cat, one can conclude from the data presented that this nucleus in Rhinolophus is homologous, on the basis of its projection pattern, to the MSO in other mammals, and it is therefore called MSO. One main proof for the homology, however, is still lacking in Rhinolophus, i.e., evidence of a direct projection from the cochlear nuclei of both sides. In Pteronotus parnellii parnellii a very well-developed MSO was found on the basis of the projection pattern from the cochlear nuclei and to the ICC (Zook, '79). The cellular arrangement is somewhat different in Pteronotus and more bipolar cells seem to be present (Zook, personal communication). With the same methods Zook and Casseday ('78) could not find a MSO in the bat Artibeus jamaicensis, which has a better-developed visual system and a larger head size than Pteronotus and Rhinolophus. According to the hypothesis of Harrison and Irving ('66), Artibeus should have a better-developed MSO than Pteronotus and Rhinolophus, which is not the case. Zook ('79) concluded from these data that bats which emit a long constant-frequency echolocation call (like Rhinolophus and Pteronotus) might have a MSO to detect the interaural time delay of the envelope of the calls. This assumption may be true, but it is somewhat contradicted by the fact that Molossus, emitting a short constant frequency, frequency-modulated echolocation call, seems to have a very well-developed MSO (personal observation). Therefore no current hypothesis can explain why one bat species has a MSO and others do not. A comparative study in different species of bats considering the anatomy of the auditory system in the context of their behavior may perhaps throw some light on these questions. A further reason why the paraolivary nucleus of microchiroptera might be homologous to the MSO comes from recent experiments of Harnischfeger ('80), who recorded from neurons in the SOC of Molossus that responded to interaural time differences as low as $10 \mu \mathrm{sec}$, indicating that bats are able to decode the low interaural time differences produced by their small head size.

\section{The nuclei of the lateral lemniscus}

In Rhinolophus and Pteronotus (Zook, '79) there are three nuclei in the projection path of the lateral lemniscus to the IC; these are the ventral (VNLL), intermediate (INLL), and dorsal (DNLL) nuclei of the lateral lemniscus. Whereas VNLL and DNLL are common in mammals, the INLL seems to be especially well developed or unique to bats.

The VNLL is a very prominent nucleus in Rhinolophus and has about the same size as the LSO. Its high development suggests important function in echolocation. In other mammals the VNLL receives fibers from the $\mathrm{CN}$ and the SOC which terminate in the ventral and dorsal parts respectively (Fernandez and Karapas, '67; van Noort, '69; Warr, '69; Browner and Webster, '75). Neurophysiological data support the idea that the VNLL must be divided into two functionally different regions, as can be expected from the connection pattern. Dorsal neurons respond to binaural and ventral neurons to monaural stimulation (Aitkin et al., '70).

As pointed out above, the INLL seems to be unique to bats. However, it is possible that this nucleus is homologous to the nucleus sagulum of other mammals and is only especially well developed in microchiroptera. This explanation would be in agreement with the findings of Adams ('79), who found a strong projection from the sagulum to the ICC in the cat. The INLL apparently has an auditory function since it projects to the ICC and receives projections from the CN and the SOC, as was dem- 
onstrated in Pteronotus (Zook, '79). Like the other two nuclei of the lateral lemniscus, the physiological functions of the INLL are unknown in microchiroptera and its seems promising to investigate the connections and the functions of the VNLL and the INLL since they are especially well developed or unique to bats.

\section{Tonotopical arrangement of projection}

The aim of the HRP experiments presented here was to obtain information on the complete afferent connections of the IC and to find out the main efferent pathways. Therefore, for the tonotopical studies only two cases were distinguished, i.e., HRP deposits into dorsal parts of the ICC and deposits into ventral regions.

The central nucleus of the IC is the main target for ascending lateral lemniscus fibers which project to the ICC in a tonotopic order. A tonotopic arrangement of neurons in the ICC, with low best frequencies localized in dorsal parts and high best frequencies in ventral parts, is a common feature in bats and other mammals. This has been shown in Myotis lucifugus and Plecotus townsendii (Grinnell, '63). Myotis lucifugus (Friend et al., '66), the cat (e.g., Rose et al., '63; Merzenich and Reid, '74; Aitkin and Moore, '75; Aitkin et al., '75; Roth et al., '78), the rabbit (Aitkin et al., '72), and the squirrel monkey (FitzPatrick, '75).

However, in the ICC of Rhinolophus a specialization has been found regarding frequency representation: The best frequencies of single units (in the range from 80 to $86 \mathrm{kHz}$ ) corresponding to the frequencies of the contant frequency component of the echolocation sounds and echoes (Schnitzler, '68) are topically overrepresented compared to other frequencies (Schuller and Pollak, '79; Pollak and Schuller, '81). These results fit well with the findings that the frequency range from 83 to $86 \mathrm{kHz}$ occupies about the same length on the cochlear basilar membrane as an octave at lower frequencies (Bruns, '76). The tonotopical organization in the ICC of Rhinolophus shows, besides the dorsoventral, a mediolateral arrangement: High-frequency neurons have been found in more medial parts of the ICC from 500 to about $2,000 \mu \mathrm{m}$ deep and in lateral parts at a depth of about 1,800 $\mu \mathrm{m}$ (Pollak and Schuller, '81). Similar results are available for the bats Myotis lucifugus (Grinnell, '63) and Molossus molossus and Molossus ater (Vater et al., 79). The fact that the frequency range of low threshold in the audiogram is overrepresented in the ICC might be a common principle in mammals. This overrepresentation is exceptionally well detectable in Rhinolophus, where the narrow-frequency range from 80 to $86 \mathrm{kHz}$ plays an important role for echolocation and is vastly overrepresented in the ICC.

Fitting the neurophysiological data with the anatomical parcellation of the ICC suggests that in the dorsolateral region of the ICC of Rhinolophus the frequency range from low frequencies up to $80 \mathrm{kHz}$ is represented and that the frequencies of the acoustical filter, i.e., the frequency region in the hearing threshold curve between 80 and $86 \mathrm{kHz}$ (Neuweiler, '70) and higher frequencies, are represented in the ventromedial region. Therefore one would expect to find labeled cells in low-frequency regions of auditory centers after HRP deposits in dorsal parts of the ICC, and in high-frequency regions after HRP deposits in the ventromedial region of the ICC.

As the HRP experiments have shown, there exist strong connections between the inferior colliculi of both sides. The data for corresponding regions on both sides suggest that there is a point to point connection between the right and left ICC and that predominantly multipolar cells give rise to the commissure of the IC. There must be a strong influence between the two inferior colliculi in the processing of auditory information, which has so far never been investigated with neurophysiological methods. The data presented show further that only a few neurons of the ICP give rise to the commissural projection and none of the ICX.

\section{Projections from the cochlear nucleus complex}

Neurons of all three subnuclei, DCN, PVCN, and AVCN, project directly to the ICC.

DCN projection: The DCN projects bilaterally to the ICC, but the projection from the contralateral side was consistently much stronger. Most projecting neurons are situated in the fusiform cell layer. This is in agreement with the findings in Pteronotus (Zook, '79), in the cat (e.g., Fernandez and Karapas, '67; van Noort, '69; Osen, '72; Adams, '79), in the rat (Beyerl, '78), and in the Rhesus monkey (Strominger, '73). The DCN projects topographically to the ICC. After dorsal IC injections labeling was found more ventrally in the DCN and after ventral IC injections cells in the dorsal and especially in the dorsocaudal unlayered region of the DCN were labeled. These facts suggest that the high frequencies are represented dorsally and the lower frequencies ventrally. This agrees with the findings that the basal turn of the cochlea projects to dorsal parts and the apical turn to ventral 
parts of the DCN in the guinea pig (Noda and Pirsig, '74) and in the kangaroo rat (Webster, 71). The DCN of microchiroptera has, in comparison to basal insectivores, the lowest progression index of all auditory nuclei (Baron, '74) suggesting that DCN does not play an important role in echolocation. In this study, however, a specialized unlayered region was detected in the dorsocaudal part of the DCN, which seems to have no homologue in other mammals. In this region labeled cells were always found when HRP was injected into the high-frequency region, i.e., the ventromedial region of the ICC.

PVCN projection: After dorsal ICC injections labeled cells were found in the contralateral ventral subdivision of the PVCN and in the lateral subdivision after ventral ICC injections. Although the cellular arrangement seems to be distinct for both subdivisions in cell-stained material, it is questionable whether they constitute functionally different subdivisions. They appear to differ only in their tonotopical arrangement with the lower frequencies represented in the ventral subdivision and the higher frequencies in the lateral subdivision. The ventral and the lateral subdivision together form an "S"-shaped convoluted structure. Such convolutions often appear in the brain when one part of a nucleus is exceptionally well developed. The overrepresentation of high frequencies in the auditory system of Rhinolophus might have led to a strong development of the lateral subdivision and therefore to the "S"-shaped structure. In all HRP-injected brains only a few labeled cells were found in the central subdivision where the "octopus cell region" might be located. This corresponds to the findings in Pteronotus (Zook, '79), the cat (Adams, '79), and the rat (Beyerl, '78), and to the findings that "octopus cells" mainly project to the periolivary nuclei and the nuclei of the lateral lemniscus, but do not have direct connections to neurons of the ICC (Warr, '69; van Noort, '69; Osen, '72; Zook, '79).

AVCN projection: The topographical arrangement of labeled cells in the AVCN after HRP injections into the IC was not as clear as in the two other cochlear subnuclei. However, in HRP injections restricted to dorsal parts of the IC (e.g., RF 18) labeled cells were found only in the anterior part of the AVCN; HRP injections into ventral parts of the IC led to heavy labeling in the entire caudal two-thirds of the AVCN. The arrangement of labeled cells is very similar to that found in Pteronotus (Zook, '79) and the cat (Adams, '79; Roth et al.,
78). It can be concluded from the data presented that the high frequency range is also highly represented in the AVCN. No labeled cells were found ipsilaterally in the AVCN in agreement with the findings in Pteronotus (Zook, '79) and the cat (Roth et al., '78) but in disagreement with findings of Adams (79) in the cat. The AVCN of microchiroptera shows, in comparison to basal insectivores, the highest progression index of the cochlear nuclei (Baron, '74), thus suggesting an important function in echolocation. The "small spherical cells" of the AVCN receive input from only two to three auditory nerve fibers, which might enable them to play an important role in fine frequency discrimination, especially if the auditory nerve fibers stem from neighboring parts of the basilar membrane. "Large spherical cells" are not present in the AVCN of Rhinolophus and other microchiroptera (Baron, '74; Poljak, '26b).

\section{Projections from the superior olivary complex}

The topographical projection pattern of the SOC to the ICC is similar to that in other mammals (e.g., Roth et al., '78; Adams, '79; Zook, 79). Whereas in the trapezoid body and the periolivary nuclei a topographical projection pattern could not be detected, the projections from the LSO and the MSO to the ICC are highly ordered. Labeled cells were found after dorsal IC injections in the lateral parts of the LSO, dorsally in the MSO, and for deep IC injections in the medial limb of the LSO and ventrally in the MSO.

\section{Projections from the nuclei of the lateral lemniscus}

In Rhinolophus the VNLL can be divided into at least two subdivisions, which were designated lateral and medial according to their location. Both subdivisions show different projection patterns. Whereas only a few cells were labeled in the medial subdivision when HRP was injected into the ICC, nearly every cell was labeled in the lateral part. A possible explanation would be that the lateral subdivision serves as a relay station for ascending $\mathrm{CN}$ fibers and that all neurons in this part project to the ICC and that neurons in the medial subdivision receive SOC fibers and project only partially to the ICC.

In every experiment labeled cells were found in the INLL. But with the large HRP deposits used in this study a topographical projection pattern of labeled cells could not be detected. 
The DNLL is poorly developed in Rhinolophus compared to other auditory nuclei and its connection pattern to the ICC is identical with that in other mammals.

\section{Projections from the auditory cortex}

In all experiments, labeled pyramidal cells were found in layer $\mathrm{V}$ of the temporal cortex. The region with labeled cells occupied a large portion of the temporal cortex, varying according to the injection site in the IC. The region where labeled cells were found covers at least the auditory cortex, which is very well developed in Rhinolophus (Ostwald, '80; Schweizer and Radtke, '80). The efferent fibers arising from pyramidal cells in the auditory cortex terminate within the pericentral and the central nucleus of the IC. Whether or not other parts of the cortex (e.g., the somatosensory cortex) contribute to this projection could not be determined in this study.

\section{Other projections}

In addition to projections from auditory nuclei to the IC, a few other projections must be mentioned here. The most consistently found "nonauditory" projections were from the dorsal column nuclei, and they were found in cases in which the ICX was affected by the HRP injection. These results agree with projections to the ICX in other mammals (Schroeder and Jane, '76; RoBarts, '79). By projections from the dorsal column nuclei to the IC, somatosensory information is apparently linked with the auditory system at the midbrain level and it seems that the ICX is not part of the direct ascending auditory pathway. Its connections to and from the ICC have not yet been investigated in Rhinolophus.

Labeled cells were found scattered in the pontine reticular formation. Most of them were concentrated in the raphe nucleus just between the two MNTB. It seems likely that connections between the reticular formation and the ICC may link arousal effects between the reticular formation and the auditory system and vice versa.

In all experiments anterograde labeling of axon terminals occurred. As expected, heavy labeling was found in the medial geniculate body which links the IC with the auditory cortex. The second main efferent IC projection is a strong projection is a strong projection from the IC to the lateral pontine nuclei. This projection has also been described in other mammals as the tectopontine tract (e.g., Moore and Goldberg, '66; van Noort, '69; Powell and Hat- ton, '69; Carey and Webster, '71; Kawamura, '75). The tectopontine tract links the auditory system, via the lateral pontine nuclei, with the cerebellum (e.g., Kawamura, '75). Neurons of the lateral pontine nuclei and of the cerebellum respond to acoustic stimulation (Boyd and Aitkin, '76; Aitkin and Boyd, '75; Jen and Schlegel, ' 80 ). A direct projection from the IC to the cerebellum, as found in Tadarida brasiliensis (Henson et al., '68) and in the cat (Powell and Hatton, '69), could not be found in $R$ hinolophus with the anterograde HRP method.

Smaller projections were found to the deep layers of the superior colliculus and the central gray matter. Both projections have to be considered very carefully because both regions are very close to the injected IC. But, the superior colliculus projection is known in other mammals (Moore and Goldberg, '66; Powell and Hatton, '69; Carey and Webster, '71; Moore et al., '77; Edwards et al., '79) and might be involved in auditory motor reflexes, such as the control of the pinna movements (Henkel and Edwards, '78). The projection from the IC to the central gray matter, which exists in many if not all mammals (e.g., van Noort, '69), may link the auditory system with parts of the vocalization system. It has been possible in bats to elicit echolocation calls with electrical stimulation of the central gray (Suga and Scblegel, '73; Suga et al., '73; Schuller, '79). A connection between auditory and vocalization systems is important for the echolocation system of Rhinolophus and other bats which change their echolocation calls according to the orientation situation. But, the poorly developed connection between the IC and the central gray disclosed by the anterograde HRP technique suggests that there must be additional links between the auditory and vocalization systems in mammals.

\section{CONCLUSION}

The data presented here have shown that the central auditory system of the greater horseshoe bat Rhinolophus ferrumequinum does not differ in general from that in other mammals. Most auditory centers (including the medial geniculate body and the auditory cortex) are especially well developed and the regions where the behavioral relevant frequencies are represented are extended in all nuclei. In contrast to earlier findings in the SOC of Rhinolophus a MSO has been defined according to its projection pattern to the IC. In the bats investigated so far an additional nucleus of the lateral lemniscus exists. 
The IC of Rhinolophus presents a very complex projection pattern. All auditory nuclei with the exception of the medial geniculate body and the medial nucleus of the trapezoid body project directly to the IC. Therefore the auditory information reaches the IC directly from the cochlear nuclei and after processing in the SOC and/or the nuclei of the lateral lemniscus and higher centers. Although the target regions of ascending fibers from different auditory nuclei do not completely overlap within the ICC, one could suggest a time delay system within the ascending auditory system so that auditory information processed at different levels reaches the IC successively. It would be of great interest to learn whether the different incoming informations are passed separately on to higher auditory centers or whether they are integrated within the IC.

\section{ACKNOWLEDGMENTS}

The author wishes to thank the members of the Arbeitskreis Neuro- und Rezeptorphysiologie for helpful discussion; doctors N. Neuweiler, O.W. Henson, Jr., G. Schuller, and M. Vater for their critical reading of the manuscript; and N. Chayegan, H. Hahn, and C. Ruehle for technical assistance. Data in this report were part of the Ph.D. thesis of the author ('78).

This investigation was supported by grants of the Deutsche Forschungsgemeinschaft, Schn. 136/6; Br. 593/2; SFB45/B22.

\section{LITERATURE CITED}

Adams, J.C. (1977) Technical considerations on the use of horseradish peroxidase as a neuronal marker. Neuroscience 2:141-145.

Adams, J.C. (1979) Ascending projections to the inferior colliculus. J. Comp. Neurol. 183:519-538.

Aitkin, L.M., and J. Boyd (1975) Responses of single units in the cerebellar vermis of the cat to monaural and binaural stimuli. J. Neurophysiol. 38:418-429.

Aitkin, L.M., and D.R. Moore (1975) Inferior colliculus. II Development of tuning characteristics and tonotopic organization in the central nucleus of the neonatal cat. $\mathrm{J}$. Neurophysiol. 38:1208-1216.

Aitkin, L.M., D.J. Anderson, and J.F. Brugge (1970) Tonotopic organization and discharge characteristics of single neurons in the nuclei of the lateral lemniscus of the cat. J. Neurophysiol. 33:421-440.

Aitkin, L.M., St. Fryman, D.W. Blake, and W.R. Webster (1972) Responses of neurons in the rabbit inferior colliculus. I. Frequency-specificity and topographic arrangement. Brain Res. 47:77-90.

Aitkin, L.M., J.L. Veale, and D.C. Crosby (1975) Inferior colliculus. I. Comparison of response properties of neurons in central, pericentral, and external nuclei of adult cat. J. Neurophysiol. 38:1196-1207.

Baron, G. ((174) Differential phylogenetic dvelopement of the acoustic nuclei among chiroptera. Brain Behav. Evol. 9:7-40.

Beyerl, B.D. (1978) Afferent projections to the central nucleus of the inferior colliculus in the rat. Brain. Res. 145:209-223.
Boyd, J., and L.M. Aitkin (1976) Responses of single units in the pontine nuclei of the cat to acoustic stimulation. Neurosci. Lett. 3:259-263.

Brown, J.C., and B. Howlett (1972) The olivo-cochlear tract in the rat and its bearing on the homologies of some constituent cell groups of the mammalian superior olivary complex. A thiocholine study. Acta Anat. 83:505-526.

Browner, R.H., and D.B. Webster (1975) Projections of the trapezoid body and the superior olivary complex of the Kangaroo rat (dipodomys merriami). Brain. Behav. Evol. $11: 322-354$.

Bruns, V. (1976) Periphera] auditory tuning for fine frequency analysis in the CF-FM bat, Rhinolophus ferrumequinum. II. Frequency mapping in the cochlea. J. Comp. Physiol. 106:87-97.

Carey, Ch.L., and D.B. Webster (1971) Ascending and descending projections of the inferior colliculus in the Kangaroo rat (Dipodomys merriami). Brain Behav. Evol. 4:400-412.

Edwards, St.B., Ch.L. Ginsburgh, C.K. Henkel, and B.E. Stein (1979) Sources of subcortical projections to the superior colliculus on the cat. J. Comp. Neurol. 184:309-330.

Elverland, H.H. (1978) Ascending and intrinsic projections of the superior olivary complex in the cat. Exp. Brain Res. 32:117-134.

Fernandez, C., and F. Karapas (1967) the course and termination of the striae of Monakow and Held in the cat. J. Comp. Neurol. 131:371-386.

FitzPatrick, K.A. (1975) Cellular architecture and topographic organization of the inferior colliculus in the squirrel monkey. J. Comp. Neurol. 164:185-208.

Friend, J.H., N. Suga, and R.A. Suthers (1966) Neural responses in the inferior colliculus of echolocating bats to artificial orientation sounds and echoes. J. Cell Physiol. 67:319-332.

Graham, R.C., and M.J. Karnovsky (1966) The early stages of absorption of injected horseradish peroxidase in the proximal tubules of the mouse kidney: Ultrastructural cytochemistry by a new technique. J. Histochem. Cytochem. 14:291-302.

Grinnell, A.D. (1963) The neurophysiology of audition in bats: Intensity and frequency parameters. J. Physiol. 167:38-66.

Harnischfeger, G. (1980) Brainstem units of echolocating bats code binaural time differences in the microsecond range. Naturwissenschaften 67:314-315.

Harrison, J.M., and M.L. Feldman (1970) Anatomical aspects of the cochlear nucleus and superior olivary complex. In W.E. Neff (ed): Contributions to Sensory Physiologie. Vol. 4. New York: Academic press, pp. 95-142.

Harrison, J.M., and R. Irving (1966) Visual and nonvisual auditory systems in mammals. Science 154:738-743.

Henkel, C.K., and St.B. Edwards (1978) The superior colliculus control of pinna movements in the cat: Possible anatomical connections. J. Comp. Neurol. 182:763-776.

Henson, O.W., Jr. (1970) The central nervous system. In W.A. Wimsatt (ed): Biology of Bats. Vol. II. New York: Academic Press, pp. 57-152.

Henson, O.W., R.P. Eager, and A.M. Henson (1968) Tectocerebellar projections in the bat, Tadarida brasiliensis murina. Anat. Rec. 160:473-474.

Illing, R.B., and H. Waessle (1979) Visualization of the HRP reaction product using the polarizing microscope. Neurosci. Lett. 13:7-11.

Irving, R., and J.M. Harrison (1967) The superior olivary complex and audition: A comparative study. J. Comp. Neurol. 130:77-86.

Jen, Ph.H.-S., and P.A. Schlegel (1980) Neurons in the cerebellum of echolocating bats respond to acoustic signals. Brain Res. 196:502-507.

Kawamura, K. (1975) The pontine projections from the in. ferior colliculus in the cat. An experimental study. Brain Res. 95:309-322. 
Masterton, R.B., and I.T. Diamond (1967) Medial superior olive and sound localization. Science $155: 1696-1697$.

Merzenich, M.M., and M.D. Reid (1974) Representation of the cochlea within the inferior colliculus of the cat. Brain Res. 77:397-415

Mesulam, M.-M. (1978) Tetramethylbenzidine for horseradish peroxidase neurohistochemistry; A noncarcinogenic blue reaction product with superior sensitivity for visualizing neural afferents and efferents. J. Histochem. Cytochem. 26:106-117.

Moore, R.Y., and J.M. Goldberg (1966) Projections of the inferior colliculus in the monkey. Exp. Neurol. 14:429-438.

Moore, J.K., F. Karapas, and R.Y. Moore (1977) Projections of the inferior colliculus in insectivores and primates. Brain Behav. Evol. 14:301-327.

Moushegian, G., A. Rupert, and M.A. Whitcomb (1972) Proeessing of auditory information by medial superior olivary neurons. In J.V. Tobias (ed): Foundation of Modern Auditory Theory. Vol II. New York: Academic Press, pp. 265-293.

Neuweiler, G. (1970) Neurophysiologische Untersuchungen zum Echoortungssystem der Grossen Hufeisennase Rhinolophus ferrumequinum, Schreber 1774. Z. Vergl. Physiol. 67:273-306.

Neuweiler, G. (1980) Auditory processing of echoes: Peripheral processing. In R.G. Busnel and J.F. Fish (eds): Animal Sonar Systems. New York and London: Plenum Press, pp. $519-548$.

Noda, Y., and W. Pirsig (1974) Anatomical projections of the cochlea to the cochlear nuclei of the guinea pig. Arch. Oto.-Rhino-Laryngol. 208:107-120.

Noort, J. van (1969) The Structure and Connections of the Inferior Colliculus. An Investigation of the Lower Auditory System. N.V., Leiden: Proefschrift, van Gorcum \& Comp.

Ollo, Ch., and J.R. Schwartz (1979) The superior olivary complex in C57BL/6 mice. Am. J. Anat. 155:349-374.

Osen, K.K. (1972) Projections of the cochlear nuclei on the inferior colliculus in the cat. J. Comp. Neurol 144:355-372.

Ostwald, J. (1980) The functional organization of the auditory cortex in the CF-FM bat Rhinolophus ferrumequinum. In R.-G. Busnel, and J.F. Fish (eds): Animal Sonar Systems. New York and London: Plenum Press, pp. 953-955.

Poljak, S. (1926a) Untersuchungen am Oktavussystem der Saeugetiere und an dem mit diesem koordinierten motorischen Apparaten des Hirnstammes. J. Psychol. Neurol. 32:170-231.

Poljak, S. (1926b) The connections of the acoustic nerve. J. Anat. 60:465-469.

Pollak, G.D. (1980) Ogranizational and encoding features of single neurons in the inferior colliculus of bats. In R. G. Busnel and J.F. Fish (eds): Animal Sonar Systems. New York and London: Plenum Press, pp. 549-587.

Pollak, G.D., and G. Schuller (1981) Tonotopic organization and encoding features of single units in the inferior colliculus of Horseshoe bats: Functional implications for prey identification. J. Neurophysiol. 45:208-226.

Powell , E.W., and J.B. Hatton (1969) Projections of the inferior colliculus in the cat. J. Comp. Neurol. 136:183-192.

RoBarts, M.J. (1979) Somatic neurons in the brainstem and neocortex projecting to the external nucleus of the inferior colliculus: An anatomical study in the opossum. J. Comp. Neurol, 184:547-566.

Rose, J.E., D.D. Greenwood, J.M. Goldberg, and J.E. Hind (1963) Some discharge characteristics of single neurons of the inferior colliculus of the cat. I. Tonotopic organization, relation of spike counts to tone intensity, and firing patterns of single elements. J. Neurophysiol. 26:294-320.

Roth, G.L., L.M. Aitkin, R.A. Andersen, and M.M. Merzen- ich (1978) Some features of the spatial organization of the central nucleus of the inferior colliculus of the cat. $J$ Comp. Neurol. 182:661-680.

Schnitzler, H.-U. (1968) Die Ultraschallortungslaute der Hufeisen-Fledermaeuse (Chiroptera-Rhinolophidae) in verschiedenen Orinetierungssituationen. $Z$. Vergl. Physiol. 57:376-408.

Schober, W. (1959) Zur Cytoarchitektur und Cytologie der Medulla oblongata der Mausohr-Fledermaus Myotis $m y$ otis (Borkhausen). Wiss, Karl-Marx Univ. Leipzig 9, Math. Nat. Reihe 5:745-771.

Schroeder, D.M., and J.A. Jane (1976) The intercollicular area of the inferior colliculus. Brain Behav. Evol 13:125-141.

Schuller, G. (1979) Vocalization influences auditory processing in collicular neurons of the CF-FM bat, Rhinolophus ferrumequinum. J. Comp. Physiol. 132:39-46.

Schuller, G., and G.D. Pollak (1979) Disproportionate frequency representation in the inferior colliculus of the Doppler-compensating Greater Horeseshoe bats: Evidence for an acoustic fovea. J Comp. Physiol. 312:47-54

Schweizer, H. (1978) Struktur und Verschaltung des Colliculus inferior der grossen Hufeisennase (Rhinolopus ferrumequinum). Thesis, J.W. Goethe Universitatet Frankfurt.

Schweizer, H., and S. Radtke (1980) The auditory pathway of the Greater Horseshoe bat, Rhinolophus ferrumequinum). In R.-G. Busnel and J.F. Fish (eds): Animal Sonar Systems. New York and London: Plenum Press, pp. 987-989.

Strominger, N.L. (1973) The origins, course and distribution of the dorsal and intermediate acoustic striae in the rhesus monkey. J. Comp. Neurol. 147:209-234.

Suga, N., and W.E. O'Neill (1980) Auditory processing of echoes: Representation of acoustic information from the environment in the bat cerebral cortex. In. R.-G. Busnel and J.F. Fish (eds): Animal Sonar Systems. New York and London: Plenum Press, pp. 589-611.

Suga, N., and P. Schlegel (1973) Coding and processing in the auditory system of FM-signal-producing bats. JASA $54: 174-190$

Suga, N, P. Schlegel, T. Shimozawa, and J. Simmons (1973) Orientation sounds evoked from echolocating bats by electrical stimulation of the brain. JASA 54:793-797

Tsuchitani, C., and J.C. Boudreau (1964) Wave activity in the superior olivary complex of the cat. J. Neurophysiol. 27:814-827.

Vater, M., P. Schlegel, and H. Zoeller (1979) Comparative auditory neurophysiology of the inferior colliculus of two Mollossid bats, Molossus ater and Molossus molossue. I. Gross evoked potentials and single unit responses to pure tones. J. Comp. Physiol. 131:137-145.

Warr, W.B. (1969) Fiber degeneration following lesions in the posteroventral cochlear nucleus of the cat. Exp. Neurol. 23:140-155.

Webster, D.B. (1971) Projections of the cochlea to cochlear nuclei in Merriam's Kangaroo rat. J. Comp. Neurol. 143:323-340.

Ziesmer, Ch. (1952) Silberfaerbung an Paraffinschnitten. Eine weitere Verbesserung der Bodian-Methode. Mikroskopie 7:415-417.

Zook, J.M. (1979) Auditory Pathways in the Brainstem of the Mustache Bat, Pteronotus p. parnellii. Thesis, Duke University.

Zook, J.M., and J.H. Casseday (1978) Auditory pathways to the tectum in Pteronotus parnellii parnellii and Artibeus jamaicensis. Bat Res. News 19;113

Zook. J.M., and J.H. Casseday (1980) Ascending auditory pathways in the brainstem of the bat, Peteronotus par nelli. In R.-G. Busnel, and J.F. Fish (eds); Animal Sonar Systems. New York and London: Plenum Press, pp. $1005-1006$ 